\title{
Experimental Studies of the Electronic Structure of Graphene
}

\author{
Aaron Bostwick ${ }^{1}$, Jessica McChesney ${ }^{1}$, Taisuke Ohta ${ }^{1,2 *}$, Eli Rotenberg1, \\ Thomas Seyller ${ }^{3}$ and Karsten Horn ${ }^{2}$ \\ ${ }^{1}$ Advanced Light Source, Lawrence Berkeley Laboratory, Berkeley, 94720 USA \\ ${ }^{2}$ Fritz Haber Institute of the Max Planck Society, 14195 Berlin, Germany \\ ${ }^{3}$ Lehrstuhl für Technische Physik Universität Erlangen-Nürnberg, 91058 Erlangen, \\ Germany
}

1.Introduction ...........................................................................................................2

2. Preparation of epitaxial graphene on silicon carbide............................10

3: Evolution of the electronic structure: from single layer to few layer

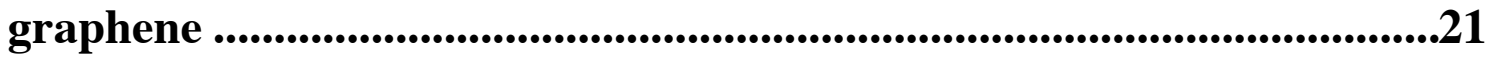

4. Gap opening and doping influence in a bilayer of graphene ................31

5. The band structure of single layer graphene: many-body effects .........40

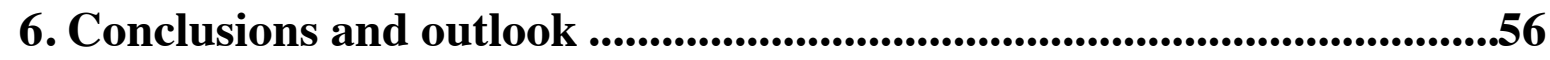

present address: Sandia National Laboratories, Albuquerque, New Mexico 


\section{Introduction}

Within the short period of about three years, graphene, a one-atom thick layer of carbon atoms arranged laterally in hexagonal "honeycomb" carbon lattice, has risen from relative obscurity, to the status of an exciting and promising model system for two-dimensional solids [1-8] . This material has many exciting and unique properties, which set it apart from most other solid materials. While the various forms of three-dimensional elemental carbon, i.e. graphite, and diamond, have been known from the earliest history, its low-dimensional forms such as zero-dimensional fullerenes and related cage molecules, and one-dimensional carbon nanotubes were only discovered within the last 25 years, and the two-dimensional form of carbon, graphene, was isolated experimentally only a few years ago. Graphene is obviously closely related to these carbon-based materials in other dimensions. The properties of graphene have been studied theoretically for about sixty years [9], and the single layer of graphene has been used as a model system in numerous theoretical studies [10-15] ; in view of the weak coupling between the graphite layers, the single layer is a reasonable starting point of theoretical examinations of graphite and other allotropes of carbon (see, for example, the book by Saito, Dresselhaus and Dresselhaus [16]). About twenty-five years ago, the study of graphene acquired a new significance when it was suggested that graphene represents a condensed matter analogue of quantum electrodynamics, in two spatial and the time dimensions [12-14]. Such theoretical considerations were not matched by experimental studies or verifications of these unusual predictions, since until very recently no preparation methods existed; indeed it was presumed that isolated graphene could not exist, as predicted by Landau for 2-dimensional solids in general in 1937 [17-19]. This has changed quite recently with the demonstration of exfoliated $[20,21]$ as well as epitaxially grown single layer graphene [22-25], and a frenzy of activities has ensued, fueled by the observation of intriguing charge transport properties, such as a high carrier mobility, a weak dependence of 
mobility on carrier concentration and temperature, and an unusual quantum Hall effect $[1,2]$ which can even be observed at room temperature [26], ten times higher than in any previous $2 \mathrm{D}$ electron system.

These results demonstrate that the nature of charge carriers is unlike those in any other solid state system. The properties arise from the structure of the graphene lattice and its electronic band structure; it results in a linear dispersion of carriers at the Fermi level, which implies that the speed of charge carriers in graphene is a constant, independent of momentum, as the speed of photons is a constant. The quantum mechanical description of the charge carriers in graphene is thus identical to the quantum mechanics of relativistic particles with a vanishingly small mass [12]. The properties of the charge carriers do not derive from their Fermi velocity, which is about $10^{6} \mathrm{~m} / \mathrm{s}$, about $1 / 300$ of the speed of light, but from the symmetry of the graphene honeycomb lattice [10]. Once the unusual transport properties of graphene were discovered, the theoretical tools to interpret the experimental data were thus already at hand, and consequently a rather comprehensive understanding of graphene's unusual physics has been gained within a short time.

Many unusual transport properties result from the fact that the carriers have a vanishing effective mass, with repercussions that go well beyond traditional solid state physics, such as carrier tunneling through a potential barrier of any height, the so-called Klein paradox [27-29]. The carriers in graphene are described by the relativistic Dirac equation, yet they are charged particles which obey Maxwell's equation, interacting through their charge - hence the name "'Dirac Fermions". It is no exaggeration to state that this discovery has opened a new chapter in solid state physics.

In order to get more insight into the electronic structure of graphene, which is the basic reason for its unusual behavior, a more detailed consideration of the band structure is important. The hexagonal lattice of graphene is shown in the top of Figure 1, with a two-atom 
basis indicated by the diamond. The lattice thus has sublattices A and $\mathrm{B}$; the 2D Brillouin zone with high symmetry points $\Gamma, \mathrm{M}, \mathrm{K}$ and $\mathrm{K}^{\prime}$ are shown below the direct space lattice. Bonding in a graphene can be compared with its building block, a single benzene ring, where the inplane bonding is due to a bonds made of the $\mathrm{p}_{\mathrm{x}, \mathrm{y}}$ orbitals, while the remaining $\mathrm{p}_{\mathrm{z}}$ orbital, perpendicular to the plane and odd under inversion, forms the $\pi$ and $\pi^{*}$ bands. The presence of two sublattices leads to two sets of bands that cross at the $\mathrm{K}$ point; the crossing is not inhibited because they belong to different irreducible representations of the space group.

A tight-binding calculation of graphene's bands B shown on the right-hand side of Figure 1; it shows the deep $\sigma$-type bands and the $\pi$ bands that cross the Fermi level at the K point, i.e. the boundary of the Brillouin zone. Near this point, the energy has a linear dependence on the wave vector, as is apparent from an enlarged section of the band structure with a small energy range and $\mathrm{k}$ vector near the $\mathrm{K}$ point. The dependence on the $2 \mathrm{D}$ wave vector $\mathrm{k}=\left(\mathrm{k}_{\mathrm{x}}, \mathrm{k}_{\mathrm{y}}\right)$ is conical as can be seen from the $3 \mathrm{D}$ rendition of the band structure in the bottom of Figure 1 .

If we denote the displacement of the wave vector from that corner point by $\delta k=k-\mathrm{K}$, for $\delta \mathrm{k} \cdot a \gg 1$, where $\mathrm{a}$ is the lattice constant, the dispersion relation is

$$
|E|=\hbar v|\partial k|
$$

where $v=\frac{1}{2} \sqrt{3} \tau a / \hbar$ is about $10^{6} \mathrm{~m} / \mathrm{sec}$, with $\tau$ the nearest neighbor hopping energy $(\sim 3$ $\mathrm{eV})$, and $a$ the lattice constant.

As mentioned above, the linear dispersion relation is not derived from a particular shape of the atomic potentials, rather, it directly stems from the symmetry of the hexagonal, two atom basis lattice of graphene (Figure 1a) as shown by Slonczewski and Weiss [10]. It implies an energy-independent group velocity of charge carriers, which thus have zero effective mass. It was noted by DiVincenzo and Mele [12] and Semenoff [13] that such particles are described by the Dirac equation of relativistic quantum mechanics, 


$$
-i \hbar v\left(\begin{array}{cc}
0 & \partial_{x}-i \partial_{y} \\
\partial_{x}+i \partial_{y} & 0
\end{array}\right)\left(\begin{array}{l}
\Psi_{A} \\
\Psi_{B}
\end{array}\right)=E\left(\begin{array}{l}
\Psi_{A} \\
\Psi_{B}
\end{array}\right)
$$

an equation that in fact goes back to McClure [30]. Here $\Psi_{\mathrm{A}}$ and $\Psi_{\mathrm{B}}$ give the amplitude of the wave function on the A and B sublattices. The differential operator couples $\Psi_{\mathrm{A}}$ and $\Psi_{\mathrm{B}}$ but not $\Psi_{\mathrm{A}}$ to itself.

Equation 2 can be written in a more compact form as

$$
v p \cdot \sigma \Psi=E \Psi
$$

with $p=-i \hbar\left(\partial / \partial_{x}, \partial / \partial_{y}\right)$ the momentum operator in the $x$-y-plane and $\sigma=\left(\sigma_{x}, \sigma_{y}, \sigma_{z}\right)$ the vector of Pauli matrices acting on the spinor $\Psi=\left(\Psi_{A}, \Psi_{B}\right)$. This spin described by the Pauli matrices is called "pseudospin" to distinguish it from the real electron spin $[15,31]$. 
a) real space

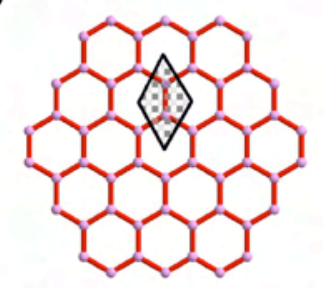

reciprocal space
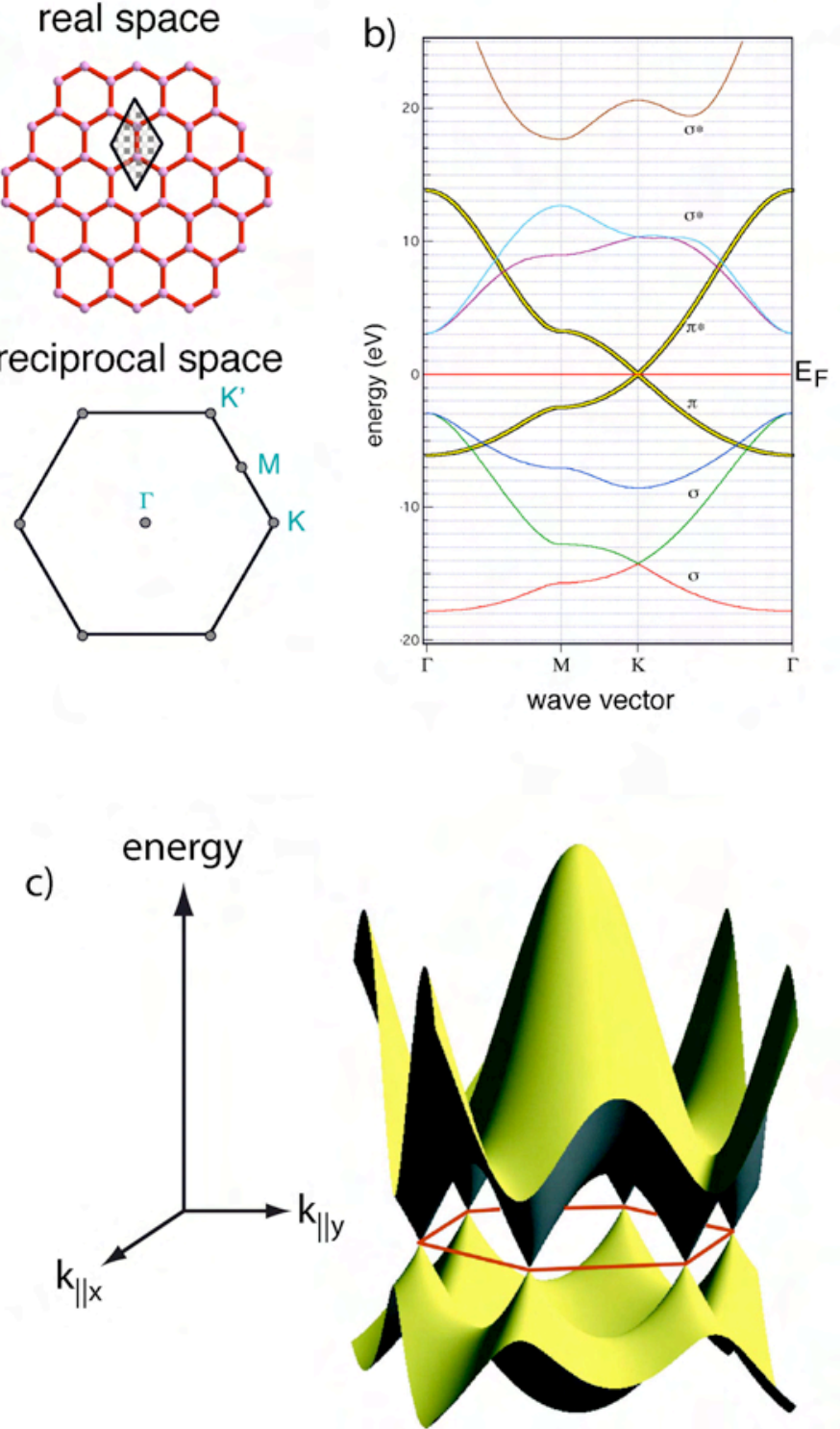

The

Figure 1: a) Lattice of graphene, with the two-atom basis as indicated by the parallelogram; the $2 \mathrm{D}$ Brillouin zone is shown below, with points of high symmetry marked. b) Tight binding band structure of graphene along the high symmetry directions. c) 3D rendition of the shape of the $\pi$ bands, showing the Dirac points where the valence and conduction bands meet at the $K$ point of the Brillouin zone). From Bostwick et al. [32]

two-dimensional Dirac equation describes states with wave vector $\boldsymbol{k}$ in the valley centered at the corner K. The valley at the opposite corner K' produces an independent set of states $\Psi=\left(\Psi_{A}^{\prime}, \Psi_{B}^{\prime}\right)$. These satisfy the same Dirac equation with $\mathrm{p}_{\mathrm{x}}->-\mathrm{p}_{\mathrm{x}}$. The dispersion relation is depicted in a 3D rendition in the bottom of Figure 1 [32]; the crossing points of the bands 
and the conical dispersion can be clearly seen. We will frequently return to the discussion of the specific properties of the graphene band structure in the following sections.

Graphene shares some of its exciting properties with carbon nanotubes, which are difficult to prepare and investigate as single entities on a large scale ensemble of identical tubes. Thus attempts were made to prepare graphene already in 2001 using epitaxial growth on silicon carbide [33] by vacuum graphitization, a process hat had been known long before to result in ordered layers of graphite with only a few layers thickness [34]. Unusual transport properties suggesting a new quantum Hall system were derived from Shubnikov-de Haas oscillations, and epitaxial growth was envisaged as a route towards graphene-based nanoelectronics [35].

The experimental breakthrough that brought experimental verification to the theoretical predictions, and has led to the intense research effort into the basic properties and applications of graphene, has been the preparation of single layer and few layer graphene (FLG) by what is termed micro-mechanical cleavage of bulk graphite; in more simpler terms, this involves rubbing a piece of graphite on a suitable substrate [20], mostly $\mathrm{SiO}_{2}$ on a silicon wafer, a rather unsophisticated technique it may seem. The decisive part then is to identify a single layer graphene flake with dimensions up to $\sim 100 \mu \mathrm{m}$ among the thousands of thicker flakes surrounding the desired sample. The important and serendipitous finding of the Manchester group was that a single layer of graphene becomes visible in an optical microscope if placed on top of a silicon wafer within a specific oxide layer in a very narrow range of thicknesses. The interference pattern produced by films even only a monolayer thick induces a faint but visible contrast [36]. This is a most fortunate situation since other methods of screening the thousands of flakes in a "rubbed" or "drawn" area on a substrate, such as electron microscopy, cannot distinguish between single layer and thicker layer films. Geim and coworkers have given detailed descriptions of ways to identify single layer graphene [36], no doubt an important help and stimulus for the rapid spread of experimental efforts and progress in the 
field. An enormous advantage of oxidized silicon as substrate is that, once the film is contacted, a field-effect device is made (using the silicon substrate as a back contact) that can be used in many different transport experiments, and even in proof-of-concept electronic devices, because it provides the possibility to vary the carrier concentration over a wide range. Moreover, the exfoliation technique permits the preparation of suspended graphene membrane, providing what the authors call "the thinnest conceivable object", offering many exciting possibilities for research [37].

The "rubbing" technique of preparing exfoliated graphene, while central to current investigations of many properties of graphene, produces samples that do not have the right properties for a number of experimental techniques though - samples are small, and the oxide substrate may be disadvantageous for probes involving charged particles. Moreover, it has been shown that graphene flakes on $\mathrm{SiO}_{2}$ exhibit a wavy mesoscopic structure [37], such that structural studies are adversely affected. Also, graphene flakes on $\mathrm{SiO}_{2}$ are not likely to be useful for large scale and general device production applications, or such experimental probes that require larger sample sizes. Epitaxial films of graphene, grown by decomposition of hydrocarbons on transition metal surfaces have been known for some time [38-43], and these show a very high structural quality $[44,45]$, but they probably cannot be used for transport studies; also, there is a likelihood of a strong interaction of the carbon $\pi$ states with the transition metal $d$ states, affecting the unique graphene band structure.

One method that does produce large scale, epitaxial and electronically decoupled graphene films had been identified well before the present interest in graphene arose $[22,34,35,46,47]$. This methods relies on the thermally induced decomposition of the top layers(s) of a silicon carbide substrate; if done in a controlled manner, this leads to a removal of the topmost silicon atoms, leaving behind a carbon layer on the silicon carbide surface in which the substrate dangling bonds are saturated, such that no strong interaction between substrate and overlayer 
occurs - see the following section for details. This and related methods probably offer a viable route towards an application of graphene in electronic devices, and with the demonstration of graphene-based transistors and other applications in exfoliated graphene [48,49], enormous efforts in thin film preparation of graphene are well under way. Epitaxial graphene on suitable substrates thus is an exciting study object, since it offers experimental access to its electronic structure by a range of techniques not applicable to graphene flakes such as photoelectron spectroscopy, but also when considering applications.

For a truly 2-dimensional systems such as graphene, which in fact happens to be a model system for a 2D solid, existing even as a freely suspended sheet of atoms [50], the application of surface-related techniques such as low energy electron diffraction (LEED), scanning tunnelling microscopy (STM) and spectroscopy (STS), valence and core level photoelectron spectroscopy etc. is a natural consequence. Not only do these techniques yield important data on the physical properties of this material, they can also be used to elucidate the growth of graphene from the initial states up to the multilayer, and the interaction between the layer and the substrate. Such studies are generally performed in an ultra high vacuum environment, i.e. under clean and controlled conditions, and using epitaxial graphene on substrates such as silicon carbide or metals $[3,33,51-55]$.

This paper is organized as follows: the introductory section with a brief motivation for research into graphene, is followed by a discussion of "surface science" aspects of the preparation of graphene films on silicon carbide in section 2. Section 3 describes the characterization of single and few-layer graphene in terms of its electronic band structure, i.e. the path from a pure $2 \mathrm{D}$ system to the quasi-2D multilayer. The special case of the graphene bilayer is discussed in section 4 , which also deals with the influence of doping on the charge distribution in the layers. The detailed shape of the bands near the degeneracy point, and the influence of many-body effects on the bands is presented in Section 5; a brief outlook 
concludes the paper.

\section{Preparation of epitaxial graphene on silicon carbide}

The graphitization of silicon carbide became known shortly after the method of producing silicon carbide was discovered by Acheson. He observed that heating of silicon carbide to very high temperatures led to the evaporation of silicon and to the formation of graphite, a useful effect for lubrication purposes [56]. Early studies of silicon carbide surfaces in ultrahigh vacuum also showed that annealing of silicon carbide surfaces at temperatures above $1100{ }^{\circ} \mathrm{C}$ leads to a depletion of silicon in the surface, leaving behind carbon-rich surfaces $[3,22,33,34,51,57-59]$ which, when processing at sufficiently high temperatures and for a sufficiently long period of time, evolves into a closed layer of graphite. Layers can be prepared from thicknesses of a fraction of a monolayer to several layers [47]. Such layers have been studied using transport methods, demonstrating that they are useful as electronic materials and may be used in electronic devices $[3,35]$. SiC possesses many polytypes; in the experiments both $6 \mathrm{H}-\mathrm{SiC}$ and $4 \mathrm{H}-\mathrm{SiC}$ material have been used as substrates. An important aspect of graphene growth on silicon carbide appears to be the structure of the starting surface [60]. Since the hexagonal surface is a polar one, there are two choices, either the siliconterminated $\mathrm{SiC}(0001)$ or the carbon-terminated $\mathrm{SiC}(000-1)$ surface. The two polar surfaces exhibit considerable differences concerning the growth of a graphene layer. On the carbonterminated surface, graphene can grow in multiple forms - in layers that are rotated $\pm 30^{\circ}$ with respect to the $\mathrm{SiC}[10-10]$ surface azimuth, or a $\pm 2.2^{\circ}$ rotation [61]; structural coherence over regions of 90 micrometer have been reported in such layers. It had been thought that multilayers of graphene on $\mathrm{SiC}(000-1)$ exhibit a high degree of azimuthal disorder because of the streaks in the LEED spots from such a surface [62]. However, it appears that this is not a 
random rotational disorder; $\mathrm{x}$-ray diffraction has shown that the streaking is due to a combination of the above orientations with respect to the substrate lattice which make adjacent islands or layers in stacks of graphene films commensurate [61]. This ordering, resulting in rotational stacking faults, then explains the puzzling result that multilayers of graphene on $\mathrm{SiC}(000-1)$ exhibit the same high mobilities as single layers, and in many respects behave as single layers. Apparently the stacking faults decouple the layers such that the symmetry-reducing action of layer stacking such as in an in-registry situation (as in graphite) does not occur.

This result may be of great significance for the use of multilayer stacks in devices; however, any kind of rotational disorder or misalignment of detrimental to use of epitaxial graphene layers in experiments that require large scale patches of graphene which are welloriented with respect to one another. Fortunately, the preparation of graphene layers by vacuum graphitization or ex-situ treatment [63] of the silicon-terminated $\mathrm{SiC}(0001)$ surface actually results in such surfaces when prepared under appropriate conditions. For the preparation of the starting surfaces, two ways for obtaining atomically flat and well-ordered surfaces exist. One method employs annealing of the surface in ultrahigh vacuum in a flux of silicon at a temperature of $950 \mathrm{C}$, whereby oxygen is removed and the silicon-rich $(3 \times 3)$ surface is obtained; at $1050 \mathrm{C}$ the $\mathrm{Si}$-rich $(\sqrt{3} \times \sqrt{3}) \mathrm{R} 30^{\circ}$ structure is then formed, which converts into the carbon-rich $(6 \sqrt{3} \times 6 \sqrt{3}) \mathrm{R} 30^{\circ}$ phase at $1150^{\circ}$. This latter phase is the important intermediate layer between the $\mathrm{SiC}$ substrate and the decoupled graphene layer as discussed in more detail below. Further annealing then leads to single and few layer graphene with $(1 \times 1)$ periodicity. The low energy electron diffraction (LEED) patterns corresponding to this sequence of preparation steps is shown in Figure 2. The second method relies on ex-situ etching of the $\mathrm{SiC}$ substrates in a stream of hydrogen $[33,35,47,55,64]$. This results in surfaces which are covered by a silicate adlayer [65]. Annealing of this surface in ultrahigh vacuum results in desorption of oxygen and the formation of the Si-rich $(\sqrt{3} \times \sqrt{3})$ R30 $0^{\circ}$ structure, 
which then transforms into the carbon-rich $(6 \sqrt{3} \times 6 \sqrt{3}) \mathrm{R} 30^{\circ}$ phase as in the first method [66].

The result of such a hydrogen etching treatment are shown in the atomic force microscopy

(AFM) images in Figure 2: the ex-situ hydrogen-etched surface is extremely flat and has

regular large scale terraces [67]. This second method is used by many groups with good

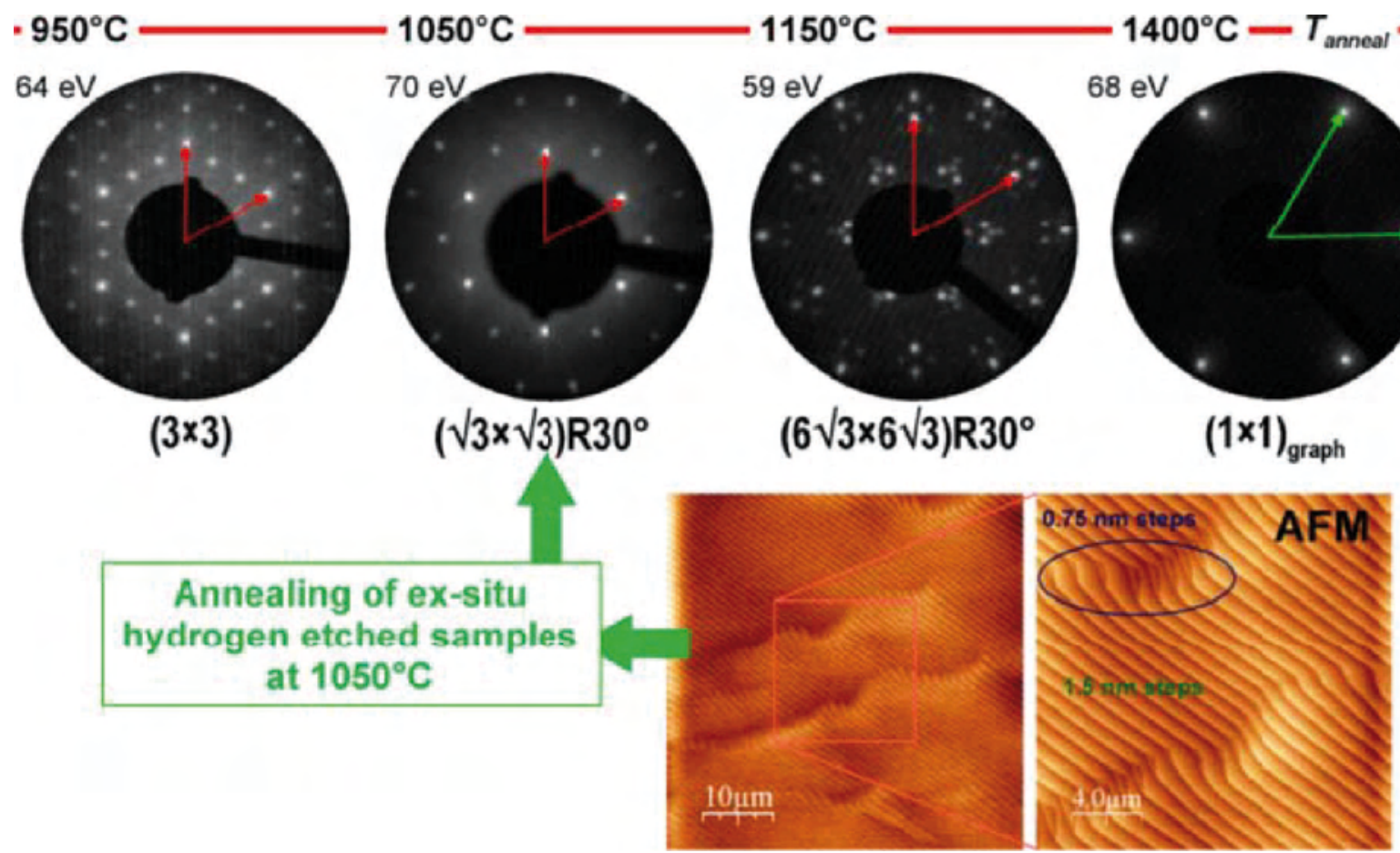

Figure 2: Typical LEED patterns observed during various stages of single and few layer graphene growth on $\mathrm{SiC}(0001)$. Annealing of ex-situ hydrogen-etched $\mathrm{SiC}(0001)$ surfaces in vacuo leads to the Si-rich $(\sqrt{3} \times \sqrt{3}) R 30^{\circ}$ structure. The effect of hydrogen etching is to remove polishing damage leading to extremely flat surfaces with micrometer sized terraces and a step height of single unit cell height, as shown by AFM. The steps are due to an unintentional miscut angle of less than 0.1 degree. From Bostwick et al. [67].

results. STM studies of graphene on the silicon-terminated $\operatorname{SiC}(0001)$ surface $[3,68-70]$ show images with large areas with clear and distinctive patterns for the single and bilayer of graphene, such that these can be readily distinguished [70]. Figure 3 a shows a constant current STM image of the graphitized $\mathrm{SiC}(0001)$ surface, which exhibits two distinct types of areas, labeled "1L" and "2L." Hexagonal reconstruction patterns with a periodicity of $17.8 \pm 2$ $\AA$ are observed in both the $1 \mathrm{~L}$ and $2 \mathrm{~L}$ regions ( the unit cell is shown in Fig. 1a). This is attributed to the $6 \times 6$ reconstruction of the $\mathrm{SiC}$ precursor layer beneath the graphene and 
shows no sign of the long range buckling observed in suspended graphene flakes [37]. The spatial frequency of $1 \mathrm{~L}$ and $2 \mathrm{~L}$ regions in the STM images can be correlated with the relative abundance of $1 \mathrm{~L}$ and $2 \mathrm{~L}$ regions measured by ARPES as explained in detail in section 3 , permitting an identification of $1 \mathrm{~L}$ terraces as monolayer graphene and $2 \mathrm{~L}$ terraces as bilayer graphene. The difference in the observed atomic structure between the single and the bilayer regions is attributed to the Bernal (A - B type) stacking of two graphene sheets on top of one another in the bilayer case, which leads to the observation of a triangular lattice, as in the case of highly oriented pyrolytic graphite HOPG, as also found by other groups. This is also consistent with recent observations from other groups [68].

Investigating the $(6 \sqrt{3} \times 6 \sqrt{3}) \mathrm{R} 30^{\circ}$ phase on silicon-terminated $\mathrm{SiC}(0001)$ is very important

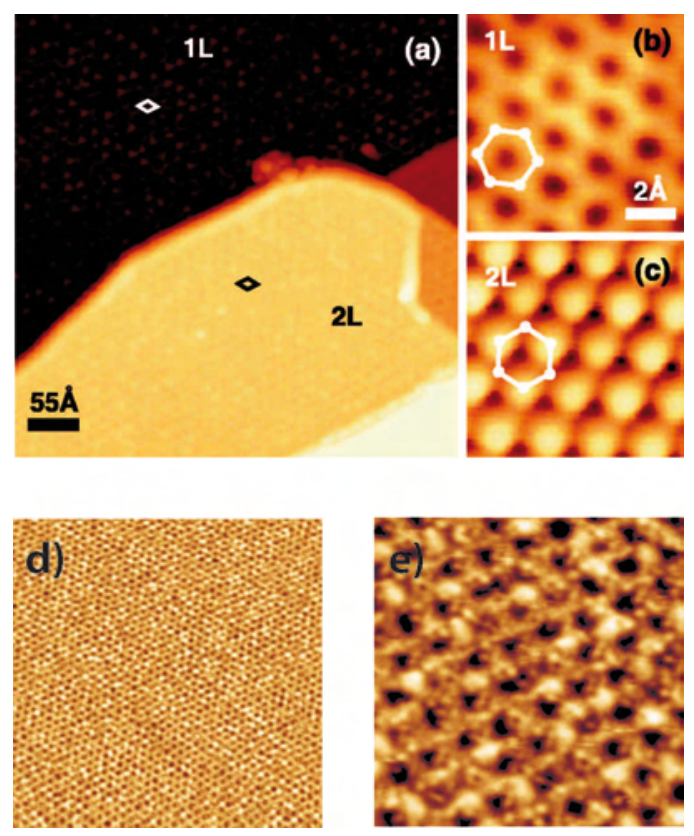

Figure 3: Constant current STM image of graphene/SiC. Monolayer and bilayer graphene regions are labeled " $L$ " and "2L," respectively. The 6 x 6 reconstruction unit cell is drawn for both types of terraces. b) and c) Constant current image showing atomic structure of monolayer and bilayer regions, respectively. Positions of carbon atoms are drawn. From Brar et al., [70]. d,e) STM images of SiC(0001) covered with 1 $M L$ graphene,taken at a large bias voltage of $U_{T=-1.85} \mathrm{~V} I_{T}=7 \mathrm{pA}$. The $z$ scale is 0.00-0.866 $\AA$ in d) and 0.00-0.83 $\AA$ in e) From Lauffer et al. [76]. 
since it is thought to electronically decouple the $\mathrm{SiC}$ surface from the graphene layer. Several models have been put forward for this phase; one of them suggests that it is a single graphene layer weakly bound by dispersion forces onto the unreconstructed $\operatorname{SiC}(0001)-(1 \times 1)$ phase [34,71] or onto the Si-rich $(\sqrt{3} \times \sqrt{3}) \mathrm{R} 30^{\circ}$ reconstruction $[22,72,73]$. The problem with a structural analysis of this surface is that its unit cell is too large to be handled by LEED surface crystallographic determination. STM images of a monolayer of graphene on SiC (0001) are shown in Figure 3d and e. Both images were obtained at a large bias voltage of $U_{T}=-1.85 \mathrm{~V}$, where no atomic resolution is observed. At such high tunneling voltages, the states contributing to the image are not the electronic states of the graphene layer, but the underlying SiC surface is imaged, [68], which is covered by the $(6 \sqrt{3} \times 6 \sqrt{3}) \mathrm{R} 30^{\circ}$ reconstruction [55], previously studied by STM. [74]. Despite the fact that the reconstruction has a $(6 \sqrt{3} \times 6 \sqrt{3}) \mathrm{R} 30^{\circ}$ periodicity, the periodicity seen in the STM images shown is $(6 \times 6)$, in agreement with previous reports $[68,70,75]$. Recently, it was shown that a careful imaging of the clean $(6 \sqrt{3} \times 6 \sqrt{3}) \mathrm{R} 30^{\circ}$ reconstruction leads to images where the true periodicity is visible [74]. It is evident from the images shown in Fig. $3 \mathrm{~d}$ and e that the reconstruction is not perfectly regular but spreads somewhat in a lateral position and an apparent height [76]. Other than that, the surface appears to be flat and homogeneous even on the large scale image shown in Fig. 3d.

Insight regarding the properties of the $(6 \sqrt{3} \times 6 \sqrt{3}) \mathrm{R} 30^{\circ}$ phase can be gained from core level spectroscopy, not only through the observation of surface core level emission which may give evidence of charge transfer between differently coordinated atoms in the surface region [77], but also since the position of the Fermi level at the surface, the amount of band bending and the Schottky barrier height may be inferred from such data. The carbon 1s core level spectrum has three components, one from bulk $\mathrm{SiC}$ and two surface-related lines $\mathrm{S} 1$ and $\mathrm{S} 2$ (Figure 4a; only S2 is indicated, S1 roughly coincides with the graphene-derived peak at $284.75 \mathrm{eV}$ ), which diminish in intensity as the graphene layers builds up. From the intensity 
behavior with excitation energy $\hbar \omega$ (i.e. change of surface sensitivity) it appears that the carbon species giving rise to the two lines S1 and S2 are located in the same layer; such data also suggest that the layer in which these atoms reside is only one atom thick. Emtsev et al. interpret the $(6 \sqrt{3} \times 6 \sqrt{3}) \mathrm{R} 30^{\circ}$ phase on the basis of these findings as a single, graphene-like layer with an intact in-plane, $\sigma$-type bonding, but in which a strong interaction with the $\mathrm{p}_{z}$ orbitals and the SiC substrate occurs [55]. This layer is devoid of states at the Fermi level and thus acts as passivation layer for the $\mathrm{SiC}$ substrate, such that subsequently growing layers exhibit properties which appear, except for the doping discussed below, practically identical to exfoliated single and few layer graphene. For a use in devices, the band alignment and transport barriers between epitaxial graphene and the silicon carbide substrate need to be known. Such information has also been obtained from core level spectroscopy $[47,78]$ for few layer graphene on $\mathrm{n}$ - and $\mathrm{p}$-type $\mathrm{SiC}$, and for $\mathrm{n}$-type for the silicon and carbon-terminated surface. Using previously gathered information about the position of the $\mathrm{C} 1 \mathrm{~s}$ core level of $\mathrm{SiC}$ with respect to the valence band maximum $\mathrm{E}_{\mathrm{v}}[47]$ one can derive the position of $\mathrm{E}_{\mathrm{F}}$ at the interface with respect to the top of the valence band $E_{v}$ as indicated by the top energy scale in Figure 4 a). This leads to the band diagrams shown in Figure 4b [55]. Since the barrier for electrons on $6 \mathrm{H}-\mathrm{SiC}(0001)$ is rather small $(0.3 \pm 0.1 \mathrm{eV})$, and the valence band offset between different $\mathrm{SiC}$ polytypes is basically zero, the authors estimate that this barrier is increased by $0.3 \mathrm{eV}$ to $0.6 \pm 0.1 \mathrm{eV}$ when using n-type $4 \mathrm{H}-\mathrm{SiC}(0001)$. The Schottky barrier is also strongly face specific [55], being much larger $(1.4 \mathrm{eV})$ on the $\mathrm{C}$-face of n-type $6 \mathrm{H}-\mathrm{SiC}$ than on the Si face, a fact which demonstrates the influence of the difference in the dipole layer on these surfaces. This could be an advantage of graphene on carbon-terminated SiC $(000-1)$ in a device context.

The nature of the electronic states of the $(6 \sqrt{3} \times 6 \sqrt{3}) \mathrm{R} 30^{\circ}$ surface becomes apparent from photoelectron spectroscopic data such as shown in Figure 4 c) and d). Seyller and coworkers have studied the changes in electronic structure in going from the $\operatorname{SiC}(0001)-(6 \sqrt{3} \times 6 \sqrt{3}) \mathrm{R} 30^{\circ}$ 
phase to the first graphene layer [55]. The photoelectron intensity maps in Figure 4 c) show the entire valence band structure of this phase, with a strongly dispersing band (marked $\sigma$ ) at binding energies from about $23 \mathrm{eV}$ to about $8 \mathrm{eV}$ below $\mathrm{E}_{\mathrm{F}}$, broken by a hybridization gap at
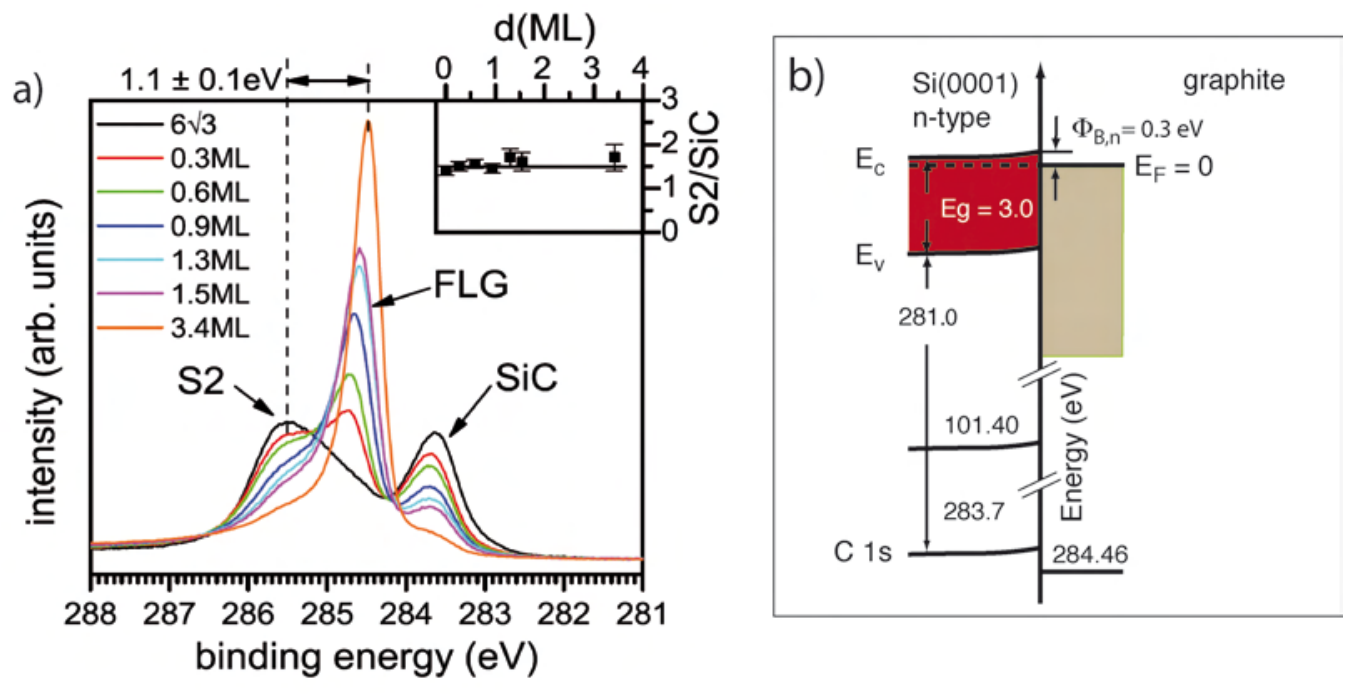
binding energy $(\mathrm{eV})$
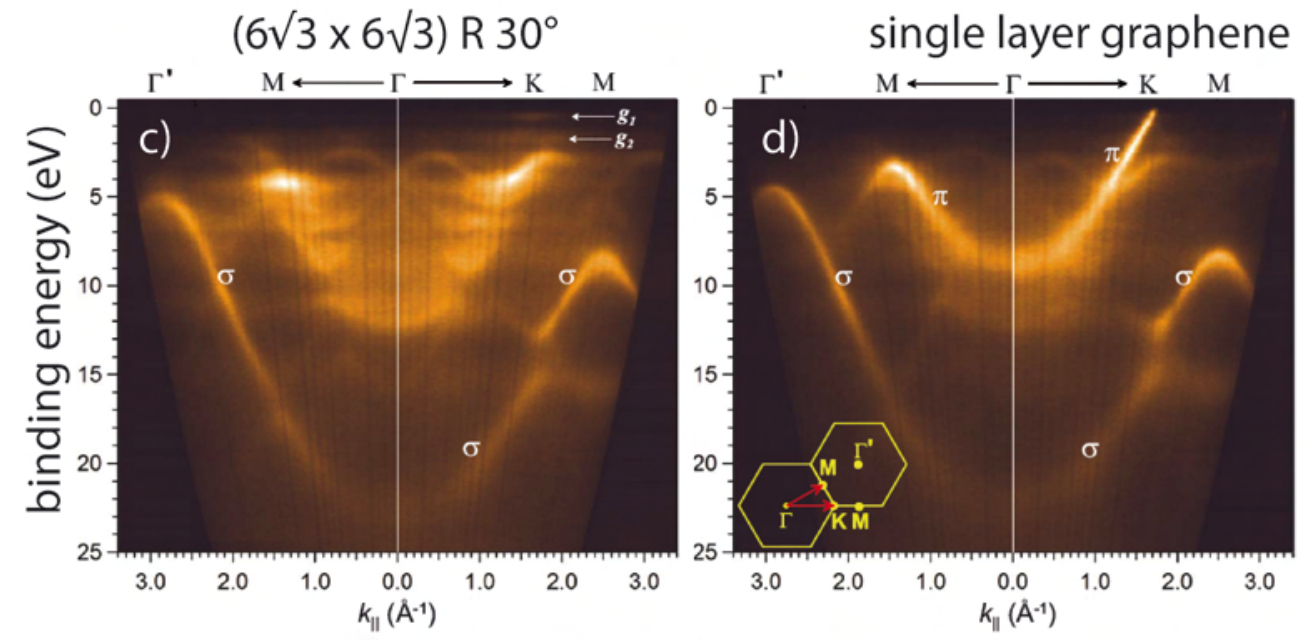

Figure 4: Photoelectron intensity map vs. binding energy and parallel momentum of (a) C 1 s core level spectra of $\operatorname{SiC}(0001)-(6 \sqrt{3} \times 6 \sqrt{3}) R 30^{\circ}$ as a function of graphene film thickness. (b) Band alignment at the SiC-graphene interface. c) angle-resolved photoemission images of the $\operatorname{SiC}(0001)-(6 \sqrt{3} \times 6 \sqrt{3}) R 30^{\circ}$ and (d) graphene on top of $\operatorname{SiC}(0001)-(6 \sqrt{3} \times 6 \sqrt{3}) R 30^{\circ}$, respectively; see text. From Emtsev. et al. [69].

$14 \mathrm{eV}$. The region from about $12.5 \mathrm{eV}$ to $2.5 \mathrm{eV}$ is dominated by emission from the bulk 
valence bands of silicon carbide, and some hardly dispersing features at 1.8 and $0.6 \mathrm{eV}$ below $E_{F}$, which are related to surface emission. The right panel (d) shows this region for a graphene layer on $\mathrm{SiC}(0001)$. The region between 23 and $14 \mathrm{eV}$ shows strong similarities with that recorded from the $(6 \sqrt{3} \times 6 \sqrt{3}) \mathrm{R} 30^{\circ}$ phase; the dispersing feature is assigned to the graphene $\sigma$ bands [54]. From the similarity in band shape the authors infer that the $(6 \sqrt{3} \mathrm{x}$ $6 \sqrt{3}) \mathrm{R} 30^{\circ}$ phase contains $\mathrm{sp}^{2}$ bonded carbon atoms with a carbon-carbon distance comparable to that in graphene. The region from about $8 \mathrm{eV}$ right up to the Fermi level exhibits the strongly dispersing $\pi$ band in the graphene layer, which is the one whose dispersion close to $\mathrm{E}_{\mathrm{F}}$ determines its transport properties. By comparison, it is obvious that a graphene-like $\pi$ band is not developed in the $(6 \sqrt{3} \times 6 \sqrt{3}) \mathrm{R} 30^{\circ}$ phase. Instead, broad structures are seen, the edge of which follows, at least over part of the range of the $\pi$ band, the expected shape, but in which zone folding effects seem to occur also. Moreover, there are no occupied states at the Fermi level. These observations suggest an interaction of at least part of the carbon $\mathrm{p}_{z}$ orbitals with the dangling bonds of the $\operatorname{SiC}(0001)$ surface in the $(6 \sqrt{3} \times 6 \sqrt{3}) \mathrm{R} 30^{\circ}$ phase. A similar behavior was predicted by theory for $\mathrm{H}$ adsorption on free standing graphene [79]. This finding is in contradiction to the proposed weak van der Waals interaction of the carbon atoms in the $(6 \sqrt{ } 3 \times 6 \sqrt{3}) \mathrm{R} 30^{\circ}$ phase $[22,34,71,73]$. Note that the $(6 \sqrt{3} \times 6 \sqrt{3}) \mathrm{R} 30^{\circ}$ phase, which exhibits a large scale "nano-mesh" with a diameter of the hexagonal subunits of about $20 \AA$ shows a considerable disorder in STM, the origin of which is not yet clear $[68,70]$. This topic is important in the context of the observed n-type doping of graphene grown on silicon carbide discussed below.

From the AFM images of the hydrogen-treated starting surface of $\mathrm{SiC}$ (Figure 2) and the STM images such as shown in Figure 3 one might conclude that the growth of graphene on silicon-terminated $\mathrm{SiC}(0001)$ leads to large, well ordered terraces. However, the large scale structural quality is limited by the lack of continuity and uniformity of the grown film [80-82]. On the Si-terminated (0001) basal plane, vacuum annealing leads to small graphene domains 
typically $30-100 \mathrm{~nm}$ in diameter. The small-grain structure is due to morphological changes of the surface in the course of the formation of the $(6 \sqrt{3} \times 6 \sqrt{3}) \mathrm{R} 30^{\circ}$ phase [83]. Moreover, decomposition of $\mathrm{SiC}$ is not a self-limiting process and, as a result, regions of different film thicknesses coexist as shown by low-energy electron microscopy (LEEM) . In Figure 5a, an AFM image of a hydrogen-treated $\mathrm{SiC}$ surface is shown, with large scale well-ordered terraces; the step direction and width (on the order of $300-700 \mathrm{~nm}$ ) of the terraces are determined by the incidental misorientation of the substrate surface with respect to the crystallographic (0001) plane. The step height is $1.5 \mathrm{~nm}$, which corresponds to the dimension of the $6 \mathrm{H}-\mathrm{SiC}$ unit cell in the direction perpendicular to the surface (c-axis). On defect-free areas of the sample, the terraces typically extend undisturbed over $50 \mu \mathrm{m}$ in length. After vacuum annealing, Fig. 5(b), the surface obviously undergoes significant modifications; it is now covered with small pits up to $10 \mathrm{~nm}$ in depth, and the original steps are hardly discernible any longer. This indicates that graphene growth is accompanied by substantial changes in the morphology of the substrate itself, leading to a considerable roughening. Because of this roughening, the graphene layer acquires an inhomogeneous thickness distribution as can be seen in the LEEM image shown in Fig.5(c). The irregularly shaped graphene islands are at most a few hundred $\mathrm{nm}$ in size. Moreover, monolayer graphene areas coexist with graphene bilayer islands as well as with uncovered regions of the $(6 \sqrt{3} \times 6 \sqrt{3})$ buffer layer.

The small domain size and inhomogeneous distribution of film thickness obviously stems from the fact that the vacuum annealing process is taking place far from equilibrium. A different method of preparation, i.e. annealing of a $\mathrm{SiC}$ substrate in an atmosphere or argon at 900 mbar has a dramatic improving influence on domain size and film homogeneity, as shown by the AFM image in Fig. 1(d). Step bunching is manifested by the formation of macroterraces. On average, their step width is larger by a factor of 5-8. The macro-steps which are running in the same crystallographic direction as the original steps increase in step height by the same factor and reach average heights of 8-15 nm. Parallel to the steps, uninterrupted 
macroterraces more than $50 \mu \mathrm{m}$ long have been observed. An analysis of the LEEM images taken with different electron energies shows that, except for narrow stripes at the edges, the large atomically flat macro-terraces are homogeneously covered with a graphene monolayer. The domain size of monolayer graphene appears to be limited by the length and width of the $\mathrm{SiC}$ terraces only. Narrower, darker regions at the downward edges of the terraces correspond to bilayer and in some cases trilayer graphene. This indicates that the nucleation of new graphene layers starts at step edges of the substrate surface.

There is a straightforward explanation for the differences between the vacuum- and argonannealed surfaces. The large roughness of the UHV annealed samples suggests that the surface is far from equilibrium [84], such that a transformation to a smooth morphology cannot be achieved under these conditions. The key factor in achieving an improved growth is the significantly higher annealing temperature of $1650^{\circ} \mathrm{C}$ that is required for graphene formation under argon at a pressure of 900 mbar as compared to $1280^{\circ} \mathrm{C}$ in UHV. Graphene formation is the result of $\mathrm{Si}$ evaporation from the substrate. For a given temperature, the presence of a high pressure of argon leads to a reduced $\mathrm{Si}$ evaporation rate because the silicon atoms desorbing from the surface have a finite probability of being reflected to the surface by collision with Ar atoms, as originally pointed out by Langmuir $[85,86]$. Indeed, in presence of the Ar atmosphere no sublimation of Si from the surface is observed at temperatures up to $1500^{\circ} \mathrm{C}$ whereas $\mathrm{Si}$ desorption commences at $1150^{\circ} \mathrm{C}$ in vacuum [63]. The significantly higher growth temperature thus attained results in an enhancement of surface diffusion such that the restructuring of the surface is completed before graphene is formed. Ultimately, this leads to the dramatically improved surface morphology. The graphene layers prepared by annealing under argon also exhibit high electron mobilities, such that the improved film quality as seen by structural methods has a direct bearing on the transport properties. For studies of the electronic structure, such as using photoelectron spectroscopy, domain sizes on the order of 30 - $100 \mathrm{~nm}$ are still perfectly useful since the domain size does not have a strong influence in 
this size range.
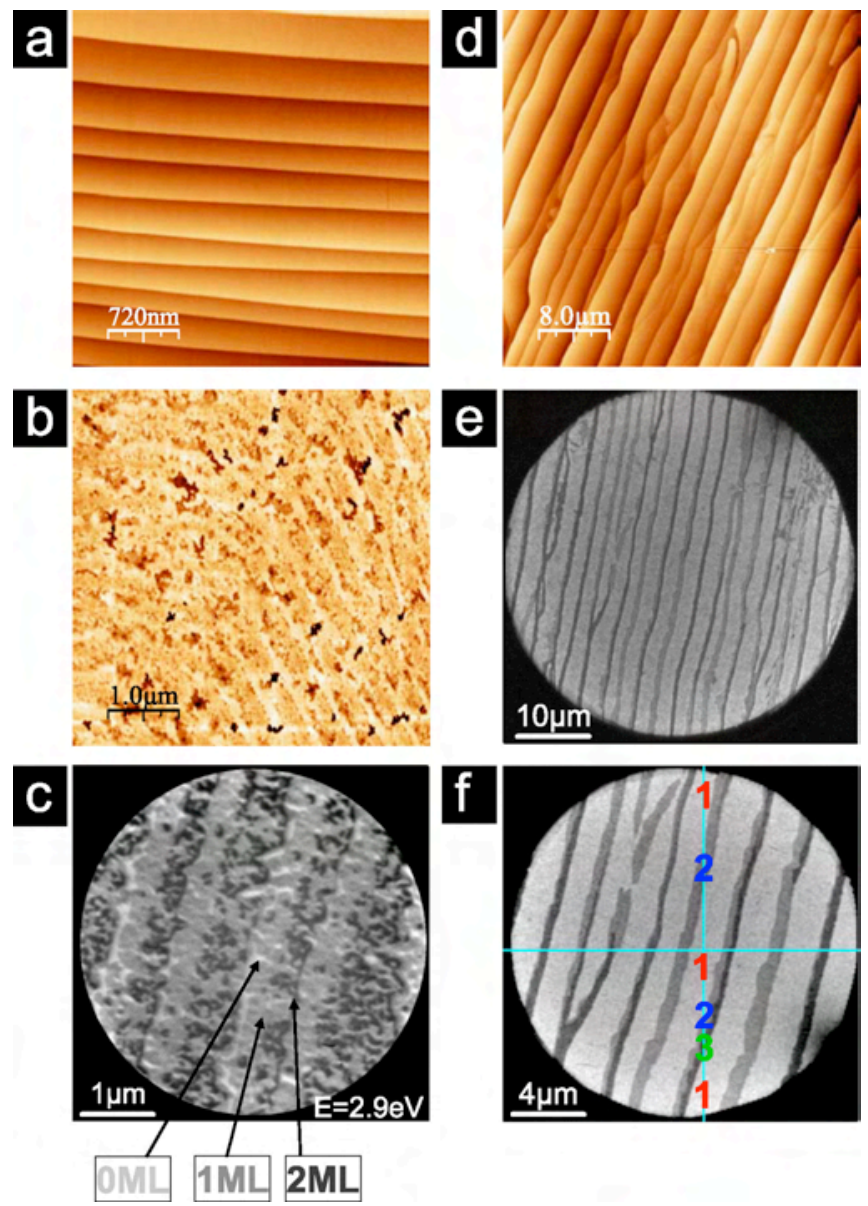

Fig 5: Evolution of silicon carbide and graphene surface morphology through different preparation methods and steps. (a) Initial surface after $\mathrm{H}$-etching imaged by AFM. The step height is $15 \AA$. (b) AFM image of graphene on $6 \mathrm{H}$-SiC(0001) with a nominal thickness of $1 \mathrm{ML}$ formed by annealing in UHV at a temperature of about $1280^{\circ} \mathrm{C}$. (c) LEEM image of a UHV grown graphene film on $\mathrm{SiC}(0001)$ with a nominal thickness of 1.2 monolayers. The image contrast is due to the locally different layer thickness. Light, medium, and dark gray correspond to a local thickness of 0,1, and 2 ML, respectively. (d) AFM image of graphene on $6 \mathrm{H}$-SiC(0001) with a nominal thickness of 1.2 ML formed by annealing in $\operatorname{Ar}$ ( $p=900 \mathrm{mbar}, \mathrm{T}=$ $\left.1650^{\circ} \mathrm{C}\right)$. (e) LEEM image of a sample equivalent to that of (d) revealing macroterraces covered with graphene up to 50 $\mathrm{\mu m}$ long and at least $1 \mu \mathrm{m}$ wide. (f) Close-up LEEM image revealing monolayer coverage on the terraces and bilayer/trilayer growth at the step edges. From Emtsev et al. [63]. 


\section{3: Evolution of the electronic structure: from single layer to few}

\section{layer graphene}

The transport properties of a single layer of graphene, a bilayer, and a system consisting of more than two layers exhibit quite considerable differences [4]. Hence a study of the evolution of the electronic structure of these systems is important. The method of graphene layer growth on silicon carbide offers a straightforward way in which to prepare these systems under clean ultrahigh vacuum conditions, with the thickness of the layers being increased by prolonged annealing times, and to examine their properties using photoelectron and other spectroscopies. Moreover, one can derive information on layer interaction and the charge distribution among the layers by a comparison between the data and band structure calculations. We will concentrate on photoelectron spectroscopic investigations since these offer a detailed insight into the electronic structure. A technical comment is appropriate here. The angle-resolved photoelectron spectroscopic images of the valence level region shown here were all recorded with the modern type of electron energy analyzer which permits to record an entire range of angles, with high energy and angular resolution. This method of recording photoemission intensities has caused a revolution in the analysis of fine detail in the electronic structure of solids $[87,88]$, for which the photoemission experiments of graphene are good example.

The photoemission "image" of the entire valence band structure of single layer graphene is shown in Figure 6 a) along principal directions of the graphene Brillouin zone (BZ) (Figure 1) [89]. For an analysis in terms of band structure calculations, let us first consider a simple single near neighbor (NN) tight binding (TB) model as presented by Saito [16] and generalized to third-nearest neighbor interaction by Reich [90]. The first-NN band structure 
can be written in closed form as

$$
E(k)=\frac{\varepsilon_{2 p} \pm \gamma_{0} w(k)}{1 \pm s_{0} w(k)}
$$

where $w(k)=\sqrt{1+4 \cos \left(\sqrt{3} a k_{y} / 2\right) \cos \left(a k_{x} / 2\right)+4 \cos ^{2}\left(a k_{x} / 2\right.}$

Here, $\gamma_{0}$ and $s_{0}$ are the nearest neighbor (NN) hopping and overlap integrals, respectively, and $\varepsilon_{2 p}$ is chosen to adjust the Fermi level $E_{F}$ to the experimental value relative to $E_{D}$. $A$ systematic fit of the bands along the principal directions is shown in Figure 6a). Since for clean graphene, the states above the Dirac energy $E_{D}$ are mostly unoccupied, the fit is only taken over the bands below $\mathrm{E}_{\mathrm{D}}$. The first-NN tight binding fit describes the overall band with reasonably well. However, the detailed curvature of the experimental bands is not well represented by this calculation, and neither are the energies of the bottom of the $\pi$ band or of the saddle point at the $\mathrm{M}$ point of the $\mathrm{BZ}$. This is because additional nearest neighbor hopping and overlap must be considered to get a more accurate fit.

Reich et al. have evaluated the band structure from first principles, and have constructed a third-NN tight binding model as a fit to the first principles calculation [90]. Their model (dotted line) is shown for comparison to the data in Figure 6(a), which has been aligned to the bottom of the $\pi$ bands at $\Gamma$. This model underestimates the observed bandwidth (below $E_{D}$ ) by around $11 \%$. A similar mismatch has also been seen for graphite, which was attributed to selfenergy effects due to electron-electron interaction [91]. In order to present a more useful tight binding fit, this band widening, and other deviations of the bands from Reich's model, are included in an empirical fit of the bands to the graphene measurements (Figure 6(a), dashed and full lines) [89]. This fit is seen to be quite satisfactory throughout a wide range of energies, and captures not only the bandwidth but also matches the detailed shape of the bands. This good agreement comes at the expense of having a rather larger hopping parameter $\left(\gamma_{0}=-5.13\right)$ than generally expected. 
Consider now the set of photoemission intensity distributions as a function of energy and wave vector kll for different thicknesses of the layer shown in the bottom part of Figure 6 [54]. The photoemission intensity distribution for the single graphene layer is shown on the bottom left-hand side. With reference to the calculated band structure (in Figure 1, and also indicated as dashed lines in Figure 6), the first thing to notice is that instead of showing two branches of bands that intersect at the degeneracy Dirac point $\mathrm{E}_{\mathrm{D}}$, only one band is visible. This phenomenon was explained by Shirley et al. by considering the transition matrix elements involved in the respective transitions [92]. Depending on the direction of $\mathrm{k}_{\|}$along which data are taken, the band that has almost no intensity in Figure 6 b) can be "switched" on or off. This effect can be put to an important use in analyzing the shape of the bands in detail. While a broadly linear dispersion predicted from the calculations is shown in the data set for the single layer in Figure 6 b), it is also obvious that there are small deviations from this shape in the region within about $200 \mathrm{meV}$ below $\mathrm{E}_{\mathrm{F}}$, and near the Dirac point; this point will be dealt with in section 5 . The patterns for a bilayer of graphene differs from that of the single layer in that a gap opens up, and two sets of $\pi$ bands occur, which is plausible because of the interaction between the layers. This happens for the three-layer situation, where three bands occur, and finally for the four-layer situation; ultimately the entire range of bands will be covered by bands in bulk graphite. The evolution of bulk bands can be followed in very fine detail here, in a similar fashion as observed in thin films of metals where quantum well states are formed from the bulk bands due to electron confinement in the direction normal to the surface $[93,94]$. The splitting between the highest and lowest $\pi$ bands increases with the number of layers, and for a four-layer film of graphene [Fig. 6(d)] it is close to that of bulk graphite, about $0.7 \mathrm{eV}$. There is a gap between the $\pi$ and $\pi *$ bands in the bilayer [Fig. 6(c)] due to the inequivalent on-site Coulomb potentials in each layer [52], an effect which will be explained in section 4. The graphene bands was modeled within a tight-binding (TB) approach by Ohta et al. to interpret the experimental $\pi$ bands in Figure $6 \mathrm{~b}-\mathrm{e}$ ). They took into 

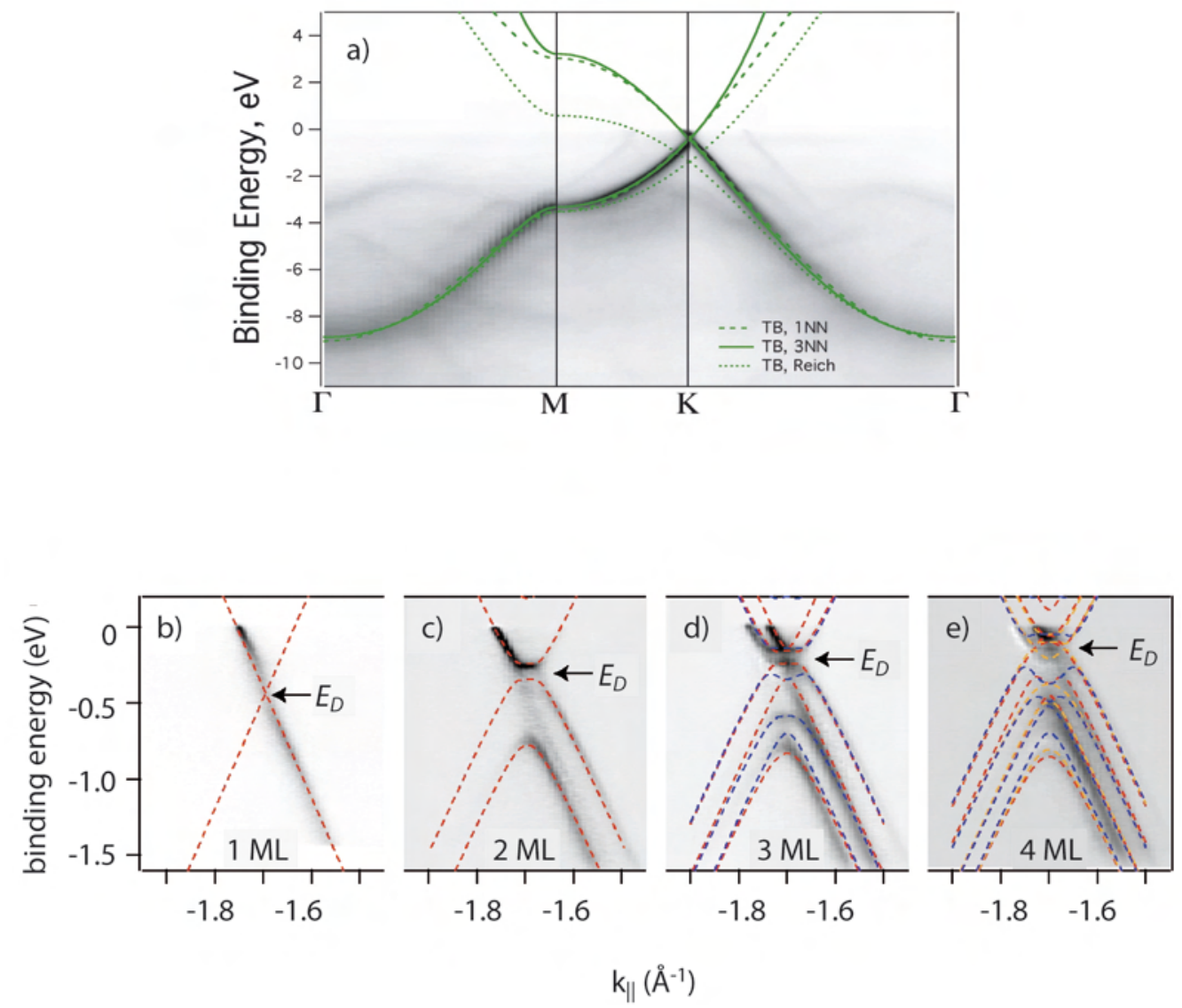

Figure 6 a) Experimental and tight binding band structures for a single layer of graphene.

The bands for the principle directions of the graphene Brillouin zone (see Figure 1) are shown for as greyscale images (darker = more intense). The best-fit tight binding bands are shown for one and three-NN models (dashed and solid, respectively). The third-NN model by Reich is shown for reference (dotted lines) From Bostwick et al., [89]. (b-e) The $\pi$ and $\pi^{*}$ bands near $E_{F}$ for 1 to 4 graphene layers, respectively. $k=1.703 \AA^{-1}$ corresponds to the $K$ point, the corner of the hexagonal Brillouin zone. The $\Gamma$ point is at $k=0 \AA^{-1}$, while the $M$ point is at $-2.55 \AA^{-1}$. The dashed lines are from a calculated tight binding band structure, with band parameters adjusted to reproduce measured bands. Red and orange lines are for Bernal-type ( $A B A B$ and $A B A C)$ stackings, while blue lines are for rhombohedral-type stackings. From Ohta et al. [54].

account the different possible stacking sequences, and different on-site potential energies, since this turned out to be important for a satisfying modeling of the data. The Hamiltonian 
was generalized from Refs. [95], [96] as

$$
H=\left(\begin{array}{cccccc}
\alpha_{1} & \beta_{0} & & & & \\
\beta_{0}^{T} & \alpha_{2} & \beta_{s} & & & \\
& \beta_{s}^{T} & \alpha_{3} & \beta_{0} & & \\
& & \beta_{0}^{T} & \alpha_{4} & & \\
& & & \beta_{s}^{T} & \cdots & \\
& & & & & \alpha_{N}
\end{array}\right)
$$

$$
\text { with } \alpha_{i}=\left(\begin{array}{cc}
E_{i} & v \pi \\
v \pi & E_{i}
\end{array}\right) \text { and } \beta_{s}=\gamma_{1}\left(\begin{array}{cc}
0 & s \\
1-s & 0
\end{array}\right)
$$

Here $E_{i}$ is the on-site Coulomb energy for layer $i, \pi=p_{x}+i p_{y}, \gamma_{1}$ is the interlayer hopping integral, $\mathrm{v}$ is the band velocity, and $\mathrm{s}=0$ for Bernal $(\mathrm{ABA} \ldots$ ) and 1 for rhombohedral (ABC . . ) stacking. The Hamiltonian operates on the layer subspace $\mathrm{i}=(1,2, \ldots \mathrm{N})$ while the $2 \times 2$ operators $\alpha$ and $\beta$ act on the $(A, B)$ sublattice sites of the same or adjacent layers, respectively. The energy scale is defined such that $\mathrm{E}_{\mathrm{F}}=0$. The Hamiltonian can be readily generalized to arbitrary stacking orders (e.g., ABAC) by suitable rearrangements of the coupling operators $\beta_{\mathrm{s}}$. It is well known that $\mathrm{ABA}$ and $\mathrm{ABC}$ stackings for graphene are energetically close, and stacking faults are commonly found in highly ordered pyrolytic graphite [97]. For samples with mixed stacking, it was assumed that the on-site Coulomb potentials do not depend on stacking sequence, and that they change monotonically across the film with the same sign [96]. Given that the potential must decay in a monotonic fashion, this considerably constrains the tight binding parameters. For trilayer graphene [Fig. 6(d)], two sets of $\pi$ bands are found, resulting from different stacking sequences. The red (light gray) and blue (dark gray) dashed lines indicate TB bands with Bernal and rhombohedral stacking, respectively. The effect of different stacking sequences is most apparent for the middle $\pi$ band near $\mathrm{E}_{\mathrm{F}}=0.5 \mathrm{eV}$, but is also seen in the upper and lower $\pi$ bands. The situation is somewhat different for the four-layer film shown in Fig. 6(e). The red and orange (light gray) dashed lines are for Bernal-type stacking, $\mathrm{ABAB}$ and $\mathrm{ABAC}$, respectively. The blue (dark gray) dashed lines are for rhombohedral-type stacking, ABCA and ABCB. Four $\pi$ bands are well 
reproduced by assuming Bernal-type stacking, with the additional weak interspersed

\begin{tabular}{|c|c|c|c|c|c|c|c|c|}
\hline $\mathbf{N}$ & $\mathbf{v}$ & n & $E_{D}$ & $E_{1}$ & $E_{2}$ & $E_{3}$ & $E_{4}$ & $\square_{1}$ \\
\hline & $10^{6} \mathrm{~m} / \mathrm{s}$ & \multicolumn{7}{|c|}{$(\mathrm{eV})$} \\
\hline 1 & 1.10 & 6.0 & -0.44 & & & & & \\
\hline 2 & 1.05 & 8.1 & -0.30 & -0.24 & & & & 0.48 \\
\hline 3 & 1.06 & 8.0 & -0.21 & -0.34 & -0.16 & -0.14 & & 0.44 \\
\hline 4 & 1.06 & 7.7 & -0.15 & -0.37 & -0.10 & -0.06 & -0.05 & 0.44 \\
\hline$\infty$ & 0.91 & & & & & & & $\sim 0.35$ \\
\hline
\end{tabular}

Table 1. Tight binding band parameters to reproduce measured band structure for $N=1-4$ layers graphene and graphite, (from Zhou et al., [62], Charlier et al [113]). The electron Idensity $n$ is measured in $10^{-3}$ electron per 2D unit cell. From Ohta [54].

photoemission intensity suggesting minor contributions from rhombohedral-type stackings. The dominance of Bernal-type stacking for a four-layer film contrasts with the coexistence of Bernal and rhombohedral stacking in the three-layer film, and suggests the role of the second nearest neighbor in stabilizing Bernal stacking in bulk graphite.

The clarity with which the number and shape of the $\pi$ bands can be determined in photoemission from graphene gives access to details of the interlayer coupling and screening effects in graphene. The bands in Figure 6 were fitted on the basis of the tight binding model above, with the results for a best fit of the bands for the parameters given in Table 1 . The resulting interlayer hopping integrals $\gamma_{1}$ for multilayer graphene are significantly larger than those of bulk graphite. In bilayer graphene, the interlayer hopping integral is reported to increase upon increasing carrier concentration [98]. This suggests that the larger interlayer hopping integral is similarly caused by the higher carrier concentrations in these layers which is caused by doping from the substrate (section 4).

The simple shape of the graphene band structure makes it possible to evaluate the total 
charge density $n$ by measuring the $\pi$ band Fermi surface areas. It was found to be almost invariant with film thickness or stacking order as shown in Table 1; this explains the observed decrease of $E_{F}-E_{D}$ with the film thickness $N$ [Figs. 6(b)-6(e)]. The deviation of the carrier concentration for different thicknesses is due to the accuracy of the fitting procedure. The extracted on-site Coulomb potentials may be used to estimate the screening length and the distribution of carrier concentrations. This is shown in Fig. 7 a), with the outermost layers' potentials aligned to the zero-reference level. The error bar is estimated from the energy width of each $\pi$ band at $\mathrm{k}_{\|}=1.65 \mathrm{~A}^{-1}$. Fitting the on-site potentials to a simple exponential decay allows a direct estimation of the interlayer screening lengths for three and four layers graphene, 1.4 and $1.9 \AA$ A respectively. The estimated screening lengths are smaller than the reported value for graphite $(3.8-5 \AA[12,99])$; however, graphite has a much smaller carrier concentration than our films, and thus a weaker electron screening. One can estimate the charge density $\mathrm{n}$ across the layers $\mathrm{N}=3$ and 4 graphene layers using Poisson's equation and the exponentially fitted potential profiles [Fig. 7 a)].

An oscillation of the carrier concentration is predicted for graphene layers [96]. However, the predicted carrier concentration oscillations are a higher-order correction to the results shown in Figure 7, considering the error bar on the experimentally determined potential profile. The carrier concentration decreases by about one order of magnitude for each adjacent layer Fig. 7 b). For the reported multilayer graphene devices [100], the screening length is expected to be larger than the present case because of lower carrier concentration; therefore, the carrier concentration profile is expected to be less steep. This suggests that the carriers in less-doped multilayers are distributed across several layers. While the electron potentials of a laterally confined surface state were previously extracted using a combination of ARPES and either scanning tunneling spectroscopy or iteratively evaluating wave functions $[101,102]$, the evaluation of the stacking order, potential, screening length, and carrier concentrations solely from ARPES band structure measurements is unique to the present study. The present analysis 
is only possible because the topology of the $\pi$ states is very sensitive to the on-site Coulomb potential, and because of the very high energy and momentum resolutions of the experiment.

Because of the simple band structure of graphene, and the fact that emission from the substrate is absent from the spectra in the region of interest, the transition from a purely $2 \mathrm{D}$ system to one that approaches the bulk structure can be followed in detail in a textbook-like fashion by measuring the dependence of photoemission intensity on the component of the wave vector normal to the surface. As expected in any true surface or $2 \mathrm{D}$ system, the $\pi$ bands of a single layer do not display a dispersion with out-of-plane electron momentum $\mathrm{k}_{\perp}$, since this is not a good quantum number (there is no interaction between atoms in the direction normal to the surface). In a layered bulk $3 \mathrm{D}$ crystal, however, the dispersion with $\mathrm{k}_{\perp}$ reflects the interaction strength between the layers. In a situation where a few layers exist, a stepwise dispersion will occur, similar to a splitting of levels in a hypothetical molecule as more atoms are added [103]. It was shown in Figure 6 b - e) that, with each added layer, an additional band occurs because of interlayer interaction. In the distribution of photoemission intensity with photon energy and hence $\mathrm{k}_{\perp}$, this is reflected in a modulation of the normalized photoemission intensity with photo-excitation energy, shown for the $\pi$ bands at a binding energy of $1 \mathrm{eV}$ below $\mathrm{E}_{\mathrm{F}}$ in Figs. $8 \mathrm{a}$ ) - d). Since there are large variations in photoemission intensity from extraneous factors such as monochromator transmission as well as cross 


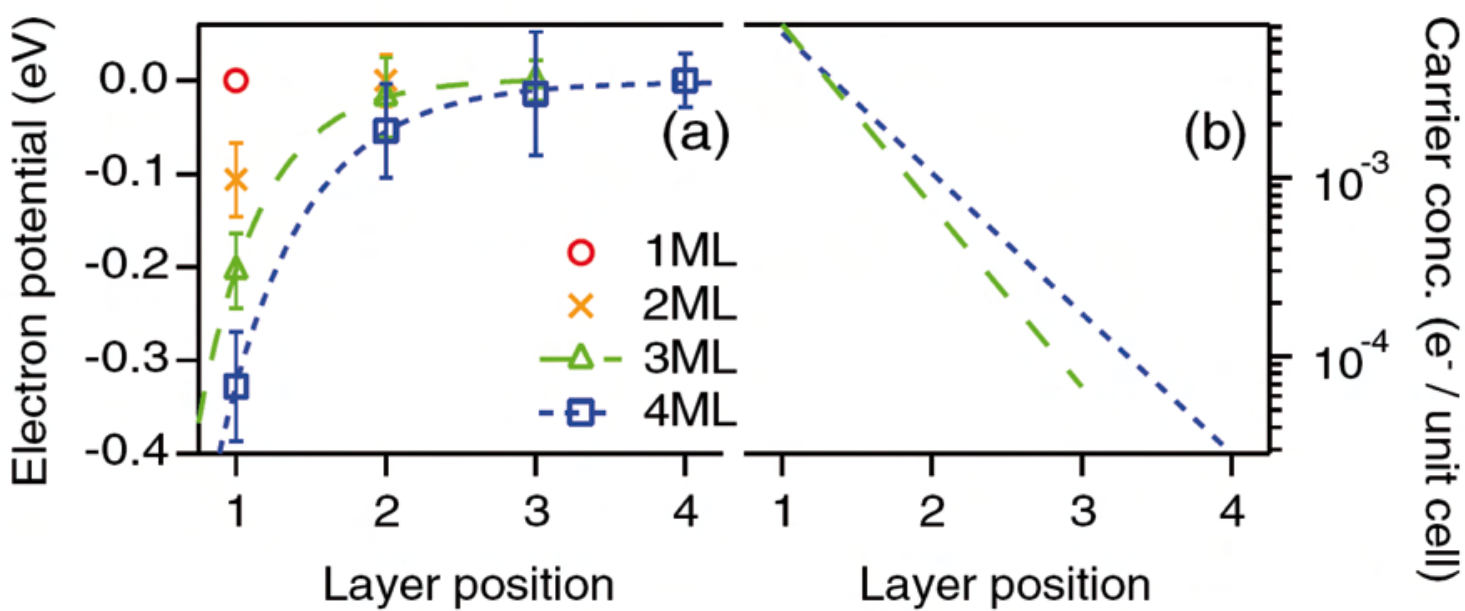

Figure 7: Potential and carrier concentration profiles of the multilayer graphene as a function of the layer positions. The electron potentials are shifted in the way that the potential of the outermost graphene layer is at zero. From Ohta et al. [54]

section changes, for the presentation in Figure 8 the photoemission intensity was normalized by dividing out the total intensity between $\mathrm{E}_{\mathrm{F}}$ and $\mathrm{E}_{\mathrm{F}}-1.5 \mathrm{eV}$ along the $\mathrm{M}-\mathrm{K}-\Gamma$ direction at each photon energy. In such a display (termed constant initial state plot), the change in intensity reflects the evolution of the transition matrix element. In these data, the normal component of the initial state electron wave vector $\mathrm{k}_{\perp}$ inside the solid was derived on the basis of the free electron final state model through $k_{\perp}=\sqrt{\frac{2 m}{\hbar^{2}}} \sqrt{E_{k i n}+V_{0}}$, with $E_{\text {kin }}$ the measured kinetic energy, an inner potential, $\mathrm{V}_{0}=16.5 \mathrm{eV}$ [104] which can be determined independently from the periodicity of the details of the dispersion in bulk graphite with photon energy, i.e. wave vector normal to the surface, and assuming that $\mathrm{k}_{\perp=} 1.703 \AA^{-1}$ at the $\mathrm{K}$ point.

The intensity pattern in Figs. 8(a)-(d), plotted as a function of $k_{\perp}$ and $k_{\|}$for the different initial states, will eventually evolve into a continuous dispersion curve as the number of layers increases toward true 3D bulk graphite. For single layer graphene, the photoemission cross section decays smoothly and monotonically [this slow variation is normalized out in the data of Fig. 8(e)]. For 2 to 4 layer graphene [Figs. 8(b)-2(d)], however, the photoemission cross sections oscillate with a periodicity of about $2.0 \AA^{-1}$. This periodicity does not match with the 
reciprocal lattice vector of bulk graphite $\left(0.932 \AA^{-1}\right)$, which includes two graphene layers in

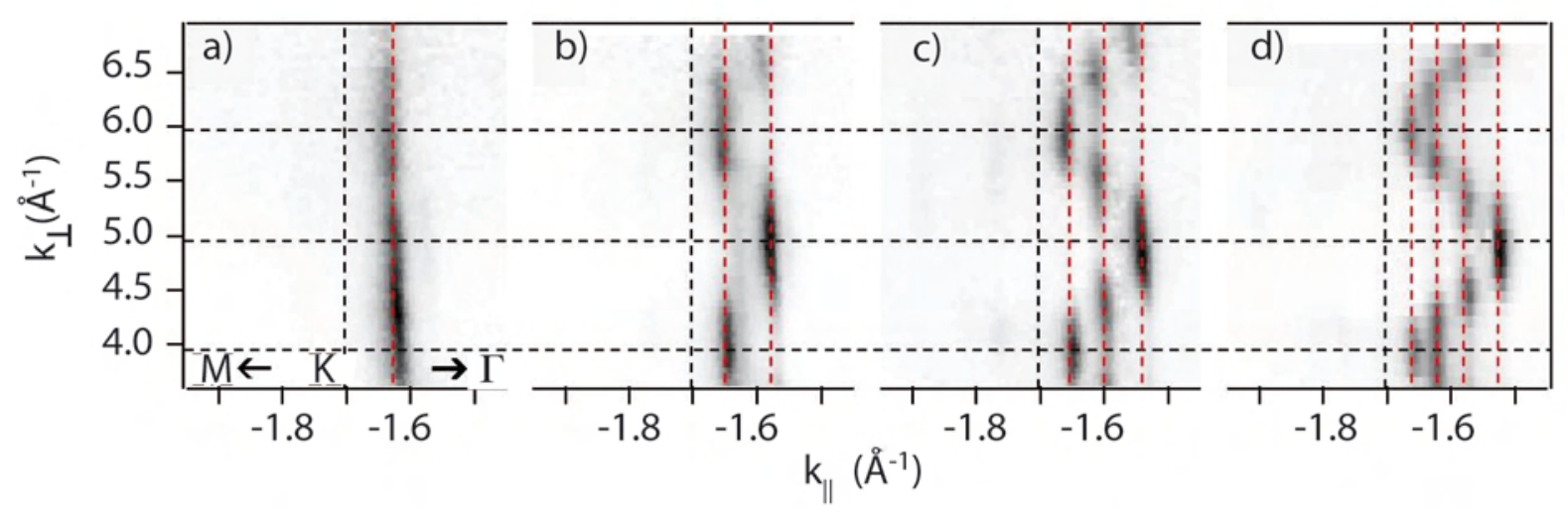

Figure 8 (a-d) Photoemission intensity oscillation of $\pi$ bands at $E_{F-1}$ eV as a function of $k_{\| \prime}$ and $k$ momentum for 1-4 layers of graphene. The photoemission intensity is normalized by the angle integrated intensity between $E_{F}$ and $E_{F-1.5} \mathrm{eV}$ for each photon energy. From Ohta et al. [54].

the unit cell, but is close to the reciprocal interlayer distance of graphite $\left(1.86 \AA^{-1}\right)$. The slightly longer periodicity of the observed intensity oscillation may also imply a reduced interlayer distance in our films, although a precise assessment of the $\mathrm{k}_{\perp}$ periodicity requires careful estimation of the inner potential. A reduction in the spacing for bilayer graphene was previously attributed to the increased screening with carrier concentration [47].

The intensity oscillations with $\mathrm{k}_{\perp}$ for $\mathrm{N} \geq 2$ are similar to oscillations reported for quantized thin film states [93] and enhanced photoemission cross section for surface states [105-107] near vertical transitions of the bulk crystal. The lack of such oscillations for monolayer graphene results not just from the trivial lack of overlying graphene layers, but also due to a notable lack of coupling to the substrate [68] consistent with the lack of $\pi$ bands in the underlying interface layer [55]. This suggests that the single layer graphene wave functions have ideal 2D character. The above evaluation is the first of its kind in succeeding to derive the influence of stacking order, layer potential screening length and carrier concentration entirely from angle-resolved photoemission experiments in any kind of layered 
system. Also unique is the observation of the transition from the pure $2 \mathrm{D}$ character of single layer graphene to an increasing influence of interlayer coupling on the path to the evolution of a 3D band structure. The deviation of the interlayer hopping integral and screening length from those of graphite, caused by the altered carrier concentrations is also an interesting observation.

\section{Gap opening and doping influence in a bilayer of graphene}

A double-layer (bilayer) film of graphene has properties that set it distinctly apart from the single-layer as well as thicker films $[95,108,109]$. This can best be seen in the transport properties which are quite different. Consider the quantum Hall effect (QHE) data in Figure 9, which shows three types of QHE behavior observed in graphene. The first one, characteristic of single-layer graphene $[1,110]$ shows up as an uninterrupted ladder of equidistant steps in the Hall conductivity $\sigma_{x y}$ which persists through the neutrality (Dirac) point, where charge carriers change from electrons to holes (Fig. 9a). The sequence is shifted with respect to the standard integer QHE sequence by $1 / 2$, so that $\sigma_{x y}= \pm 4 e^{2} / \mathrm{h}(\mathrm{N}+1 / 2)$ where $\mathrm{N}$ is the Landau level (LL) index and factor 4 appears due to double valley and double pseudospin degeneracy of graphene. This unusual ,half-integer“ sequence is now well understood as arising from the QED-like quantization of graphene's electronic spectrum in a magnetic field $[111,112]$. The existence of a quantized level at zero energy, which is shared by electrons and holes [Fig. 4c)], is essentially everything one needs to know to explain the anomalous QHE sequence. Bilayer graphene exhibits an equally anomalous QHE [Fig 4b)] [108]; here, the standard sequence of Hall plateaux $\sigma_{x y}= \pm N 4 e^{2} / h$ is found, but the first plateau at $\mathrm{N}=0$ is missing, which also implies that bilayer graphene remains metallic at the neutrality point. The origin 

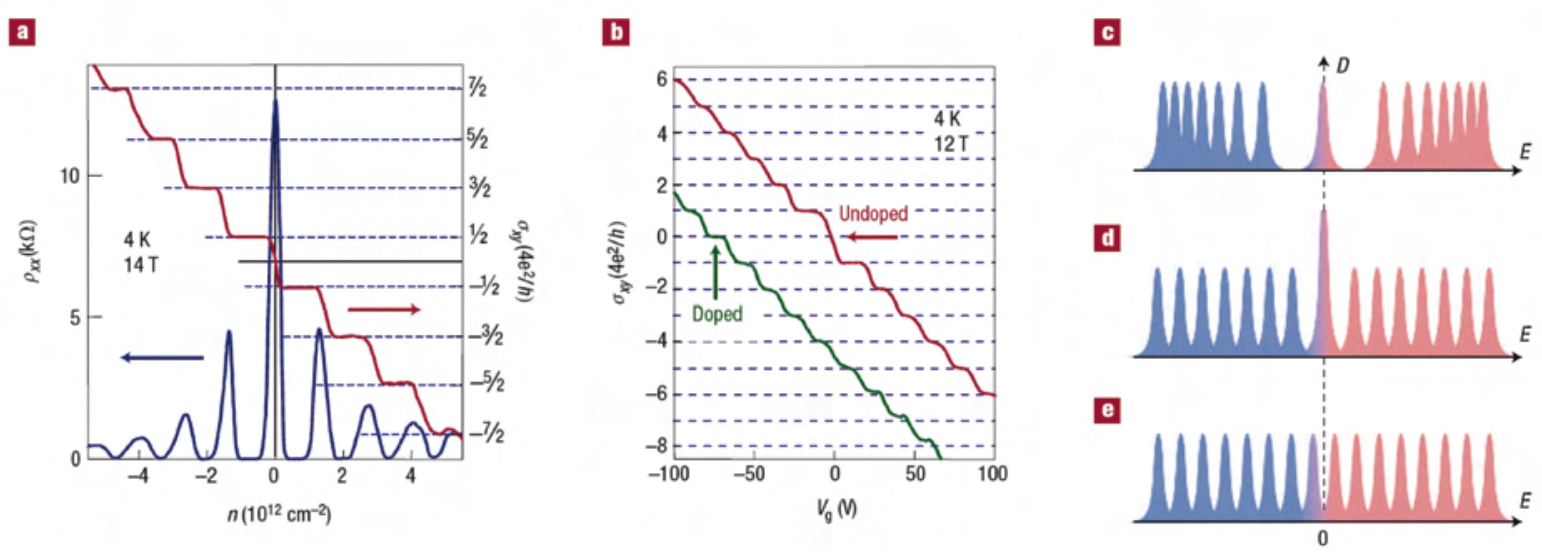

Figure 9 a) The hallmark of massless Dirac fermions is that QHE plateaux in $\sigma_{x y}$ exist at half integer values in terms of $4 e^{2} / h$ (from Geim and Novoselov [4]. b) In the anomalous QHE for massive Dirac fermions in bilayer graphene, $\sigma_{x y}$ exhibits the standard QHE sequence with plateaux at all integer $N$ of $4 e^{2} / \mathrm{h}$ except for $N=0$. The missing plateau is indicated by the red arrow. The zero- $N$ plateau can be recovered after chemical doping, which shifts the neutrality point to high $V_{g}$ so that an asymmetry gap (about $0.1 \mathrm{eV}$ in this case) is opened by the electric field effect (green curve). $c-e$, Different types of Landau quantization in graphene. The sequence of Landau levels in the density of states $D$ is described by $E_{N} \propto \sqrt{ } N$ for massless Dirac fermions in single-layer graphene (c) and by $E_{N} \propto \sqrt{ } N(N-1)$ for massive Dirac fermions in bilayer graphene (d). The standard Landau level sequence $E_{N} \propto N+1 / 2$ is expected to recover if an electronic gap is opened in the bilayer (e).

of this anomaly lies in the nature of quasiparticles in bilayer graphene, which are chiral, similar to the massless Dirac fermions in the single layer, but have a finite mass $m \approx 0.05 \mathrm{~m}_{0}$. The Landau quantization of 'massive Dirac fermions' gives rise [109] to an additional degeneracy which leads to the missing zero-E plateau and the double-height step in Fig. 9 b). Finally, the normal integer QHE can be recovered in bilayer graphene by inducing a difference in the two layers, either through the electric field effect [Fig. 9b], in which the gate voltage not only changes the charge in the layers, but also induces an asymmetry between the two graphene layers, which results in a semiconducting gap $[95,113]$, or through selective doping of one of the layers [52]. The resulting asymmetry eliminates the additional degeneracy of the zero energy Landau level and leads to the "normal" integer QHE sequence by splitting the double step into two (Fig. 9e). The electronic band structure changes 
significantly via the electric field effect, and the semiconducting gap can be tuned continuously from zero to $0.3 \mathrm{eV}$ [108]. It is then interesting to study the electronic structure of the bilayer in detail, and to examine the influence of additional doping.

The difference in the valence band structure of single and multilayer graphene was already discussed in section 3 , in terms of additional band formation beyond the single layer. As shown below, photoemission experiments on bilayer graphene can also be used to study the influence of layer charge concentration, through electron doping by "chemical" means, and by this method the band structure near the Dirac point can be modified. Let us first consider the influence of layer stacking and symmetry on the band structure of the bilayer in a model (Figure 10). The graphene band structure is sensitive to the lattice symmetry. If the hexagonal layer structure is composed of nonequivalent elements, such as in boron nitride, the lateral, inplane symmetry is broken, resulting in the formation of a large gap between $\pi$ and $\pi^{*}$ states [114]. The symmetry can also be broken with respect to the c axis by stacking two graphene
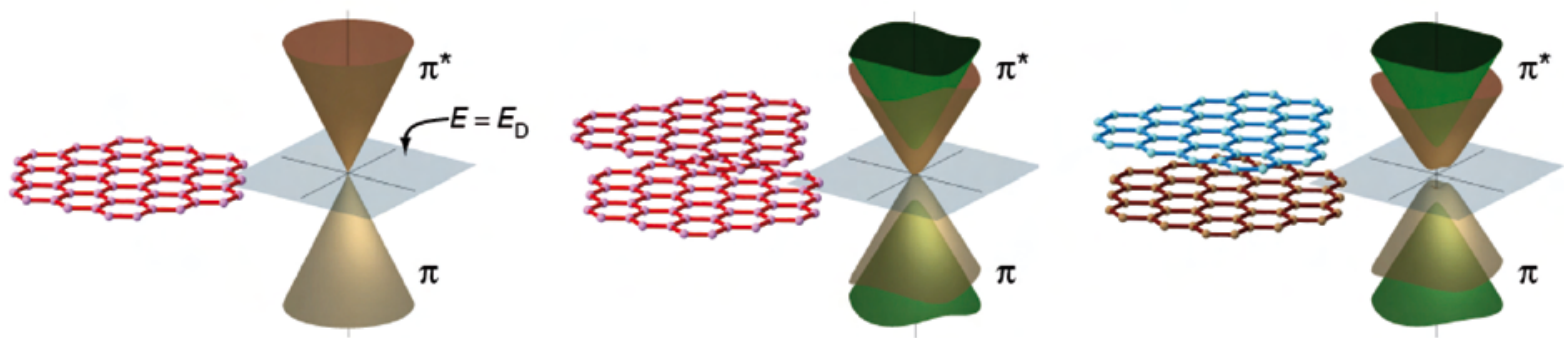

Figure 10: Electronic structure of a single (A), symmetric double layer (B), and asymmetric double layer $(C)$ of graphene. The energy bands depend only on in-plane momentum because the electrons are restricted to motion in a two-dimensional plane. The Dirac crossing points are at energy $E_{D}$. From Ohta et al. [52].

layers in Bernal stacking (the stacking fashion of graphite) as suggested by McCann and Fal'ko [109][Fig. 10 b)]. Because the unit cell of a bilayer contains four atoms, its band structure acquires two additional bands, $\pi$ and $\pi^{*}$ states, in each valley split by interlayer (AB) coupling, and two lower energy bands. If the individual graphene layers in a bilayer are rendered inequivalent [Fig. $10 \mathrm{c}$ )], then an energy gap between low-energy bands forms at the 
former Dirac crossing point. Provided that the charge state is such that the Fermi level lies within the gap, a semimetal-to-insulator transition occurs (as an interesting aside, if this symmetry breaking could be controlled externally, the electronic conductivity would change through this transition, suggesting that a switch with a thickness of two atomic layers could be constructed).

This effect of layer inequivalence on the band structure can be measured using angleresolved photoemission from bilayer graphene. Because the SiC valence band edge lies about $2.6 \mathrm{eV}$ below $\mathrm{E}_{\mathrm{F}}$ and the conduction band edge about $0.4 \mathrm{eV}$ above $\mathrm{E}_{\mathrm{F}}$ (Figure 4, [78]), the $\mathrm{SiC}$ states are well separated from both $\mathrm{E}_{\mathrm{F}}$ and $\mathrm{E}_{\mathrm{D}}$, and the bilayer graphene states can be regarded as practically decoupled from the substrate. The symmetry of the bilayers is then broken by the dipole field created by the difference in charge in the interface region of the top $\mathrm{SiC}$ and $(6 \sqrt{3} \times 6 \sqrt{3}) \mathrm{R} 30^{\circ}$ layer and the accumulation of charge on the first graphene layer next to the interface, rendering the two graphene layers inequivalent with respect to charge and electrostatic potential. We can induce further n-type doping by the depositing alkali metal atoms, in this case potassium, onto the second graphene layer on the vacuum side, which donate their lone valence electrons to the surface layer, forming another dipole. These surface and interface dipole fields act as the symmetry-breaking factor, which controls the presence or absence of the gap at the crossing energy $\mathrm{E}_{\mathrm{D}}[$ Fig. $10 \mathrm{~b}$ and $\left.\mathrm{c})\right]$. The net dipole field between the two graphene layers results from the short screening length (about $4 \AA$ ) along the c axis, which is comparable to the layer thickness (3.4 $\AA$ ). A similar charge localization has been observed at the surface of graphite and graphene multilayers in an externally applied field $[100,115]$. The binding energy - momentum dispersion relation of $\pi$, $\pi^{*}$, and $\sigma$ states along high-symmetry directions measured by ARPES in Figure 11 exhibits a $\sim 0.4$-eV splitting of the $\pi$ state, confirming that the sample is composed predominantly of two graphene layers; as shown in studies of film morphology, graphene on silicon carbide exhibits a mixture of layer thicknesses, with one being dominant, a process that can be optimized through a careful 
annealing process [81]. The constant-energy contours in momentum space of $\pi$ and $\pi^{*}$ states near $E_{F}$ (Fig. $11 \mathrm{D}$ to $\mathrm{F}$ ), show the presence of the electron and hole pockets above and below
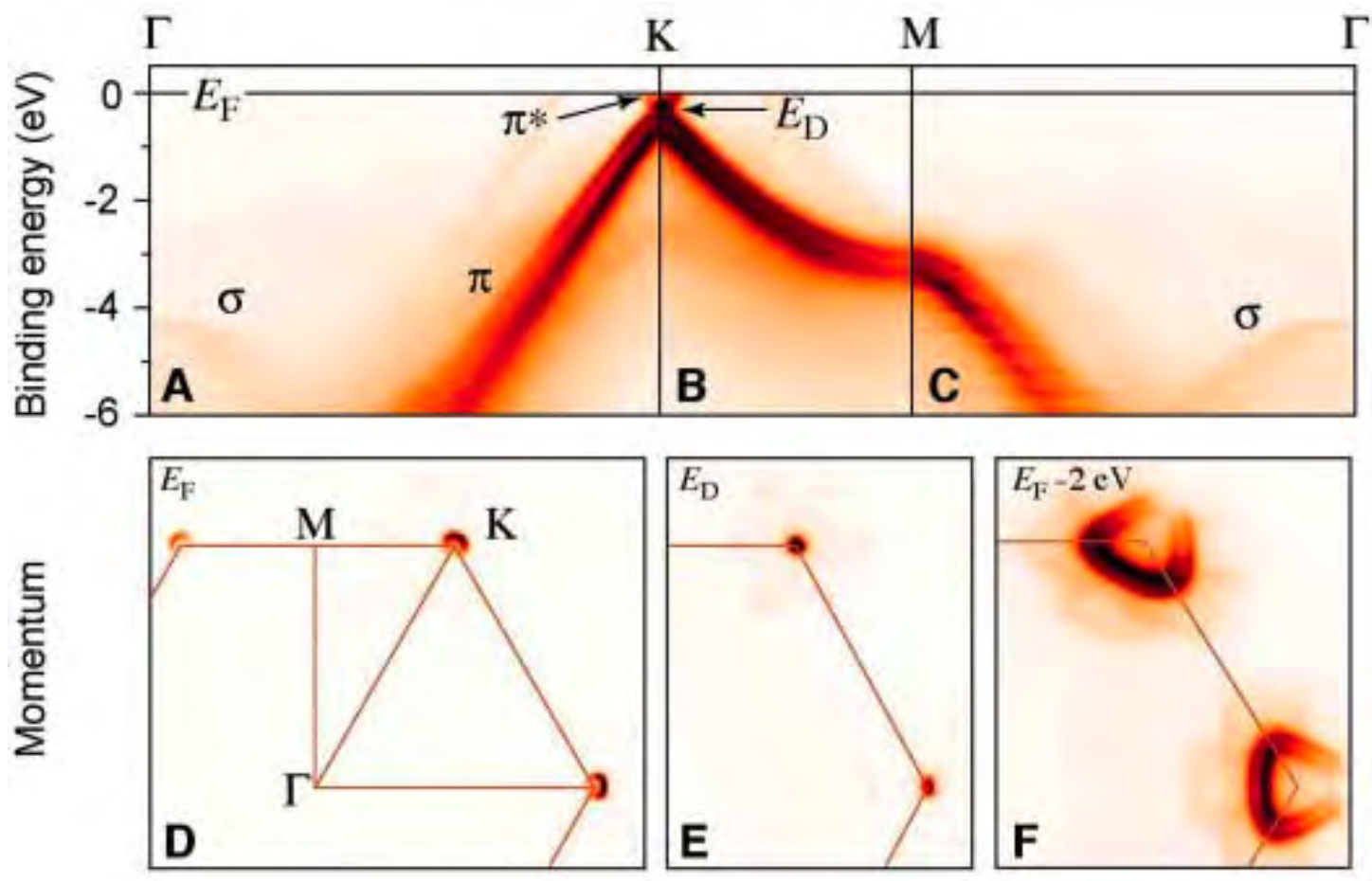

Figure 11: Energy-momentum disperSibpmelatibm of $p, p^{*}$, and s states of bilayer graphene. ( $A$ to $C$ ) Energy-momentum dispersion along high-symmetry directions. ( $D$ to $F$ ) Constant energy contours at $E_{F}, E_{F}-0.4 \mathrm{eV}=E_{D}$, and $E_{F}-2 \mathrm{eV}$. The high symmetry points, directions, and Brillouin zone boundaries are indicated in (D). From Ohta et al., [54].

the Dirac point, which can be seen here because the n-type doping pulls $E_{D}$ below the Fermi level. In the constant energy plots, six weak replicas of the $\pi$ and $\pi^{*}$ states surrounding the primary states can be seen, as (rather faint) points around the upper spot in Figure $11 \mathrm{E}$, and as roughly triangular shapes in Fig. $11 \mathrm{~F}$, arranged in a hexagonal manner. As shown in Figure 2, low energy electron diffraction shows that graphene layers grown on the $\mathrm{SiC}$ substrate display a nearly commensurate superstructure with relative lattice constant $6 \sqrt{ } 3 \times 6 \sqrt{3}$ rotated by $30^{\circ}$ with respect to the substrate because of the difference between the graphene lattice constant of $2.46 \AA$ and that of $\mathrm{SiC}, 3.07 \AA$ [22]. The replicas of the $\pi$ and $\pi^{*}$ states are brought about by scattering off of this superstructure in a fashion similar to those in other nearly incommensurate systems. What is also apparent in Figure $11 \mathrm{~F}$ is the deviation from the 
conical dispersion, the so-called trigonal distortion or warping, at an energy well below the Dirac point.

The effects of doping the bilayers are shown in Fig. 12, which compares the as-prepared film to coverages of potassium. There is a rigid shift of the $\pi$ and $\pi^{*}$ states (investigated in detail by Kihlgren et al. [116], who showed that his also applies to the $\sigma$ bands) towards higher binding energy because of an increased carrier concentration. The upper unoccupied $\pi^{*}$ state drops below $\mathrm{E}_{\mathrm{F}}$ at a charge density of 0.0125 electron per unit cell and continues dropping down with higher potassium coverage; because of the simple band structure of graphene, the electron carrier densities of each stage can be determined from the relative sizes of the Fermi surfaces with respect to the surface Brillouin zone of graphite. Plotted next to the intensity maps are calculated tight-binding bands (solid lines) [109], where the low-lying electronic states near the $\mathrm{K}$ point of the Brillouin zone are described by the solution of a simple 4 x 4 Hamiltonian as $\varepsilon_{a}(k)= \pm\left[\frac{\gamma_{1}^{2}}{2}+\frac{U^{2}}{4}+\left(v^{2}+\frac{v_{3}^{2}}{2}\right) k^{2}+(-1)^{a} \sqrt{\Gamma}\right]^{\frac{1}{2}}(8)$, where the band index $\alpha=1,2$ and $\Gamma=\frac{1}{4}\left(\gamma_{1}^{2}-v_{3}^{2} k^{2}\right)^{2}+v^{2} k^{3}\left(\gamma_{1}^{2}+U^{2}+v_{3}^{2}\right)+2 \gamma_{1} v_{3} v^{2} k^{3} \cos 3 \phi q(9)$ and $-v_{3}=\sqrt{3} \alpha \gamma_{3} / 2 \hbar(10)$.

Here $\mathrm{k}$ is the momentum in $\AA^{-1}, \phi$ is the azimuthal angle, $v$ is the band velocity (m/s x $\hbar \mathrm{x}$ $\left.10^{10}\right), \mathrm{U}$ is the difference in the onsite Coulomb potentials of the two layers, $\gamma_{1}(\mathrm{eV})$ and $\gamma_{3}$ $(\mathrm{eV})$ are out-of-plane nearest-neighbor and next-nearest neighbor interaction parameters, and a $(\AA)$ is the graphite lattice constant. These are adjusted to reproduce the measured band structures over a large energy range. $\mathrm{U}$ is chosen to match the gap at the $\mathrm{K}$ point. The most important observation in the data in Figure 12 is the variation in the apparent gap at the $\mathrm{K}$ 
point: there is a clear gap in the clean bilayer spectrum, which closes at higher doping, and finally opens again. This gap variation is reproduced by the tight-binding calculation and is attributed to the variation in the relative potentials of the two layers as schematically shown in the bottom of Figure 12: initially, the first graphene layer near the interface has a larger carrier density than the top (surface) layer. At intermediate coverages of potassium, charge transfer to the surface layer causes the charge densities to be equal, such that the gap closes, as schematically shown in Figure 10. At higher coverages of potassium, the surface layer now has a higher carrier concentration such that the layers become inequivalent again, leading to a reopening of the gap. Away from the K point, the gap is generally smaller than the prediction because the cusps extending into the gap between the $\pi$ and $\pi^{*}$ bands are much sharper than in the model. As a result, the gap for the uncovered film is not clearly resolved, although a shift of the bands is readily apparent from the flattening of the $\mathrm{p}^{*}$ band edge and the lack of spectral weight at $E_{D}$. Sufficient asymmetry is developed for higher doping that the gap is unambiguously open.

Data from many different coverages are combined in the display of photoemission spectra at the K point as a function of doping, in order to systematically follow the evolution of the gap between $\pi$ and $\pi^{*}$ states [Fig. 13a]. The blue markers are the positions of the tight-binding $\pi$ and $\pi^{*}$ bands. The data and calculated energies of the $\pi$ and $\pi^{*}$ states clearly display the closing and reopening of the gap. The yellow line is the energy difference $E_{F}-E_{D}$, which increases by about $0.32 \mathrm{eV}$ with respect to the as-grown sample, reflecting the overall doping level of the film. The modeling of the experimental data on the basis of the tight-binding offers the interesting possibility to study the influence of carrier concentration; this is shown in Figure 13B, where the Coulomb potential difference $U$ between the two layers and the nearest neighbor interaction parameter $\gamma_{1}$ are shown as a function of doping level. U displays a sign change at the electron concentration where the gap closes. It is expected that $\mathrm{U}$ increases with an increase of the charge difference in either graphene layer, induced by the 
fields at the respective interfaces. The potential of each graphene layer is estimated from Poisson's equation, based on the Schottky barrier height of $0.4 \mathrm{eV}$ [78], assuming infinitely thick graphene multilayers. For the as-prepared sample, the potential difference between the first and second layers shows reasonable agreement with the Coulomb potential difference U estimated from the size of the gap evaluated in the tight-binding model. A monotonic increase is seen in $\gamma_{1}$, which measures the interlayer interaction as a function of electron concentration in both layers. This suggests that at higher electron density, the overlap between orbitals of adjacent graphene layers increases. This may be due to the smaller interlayer distance caused by a shorter screening length. By controlling the carrier density in a bilayer of graphene, the occupation of electronic states near $\mathrm{E}_{\mathrm{F}}$ and the magnitude of the gap between the valence and

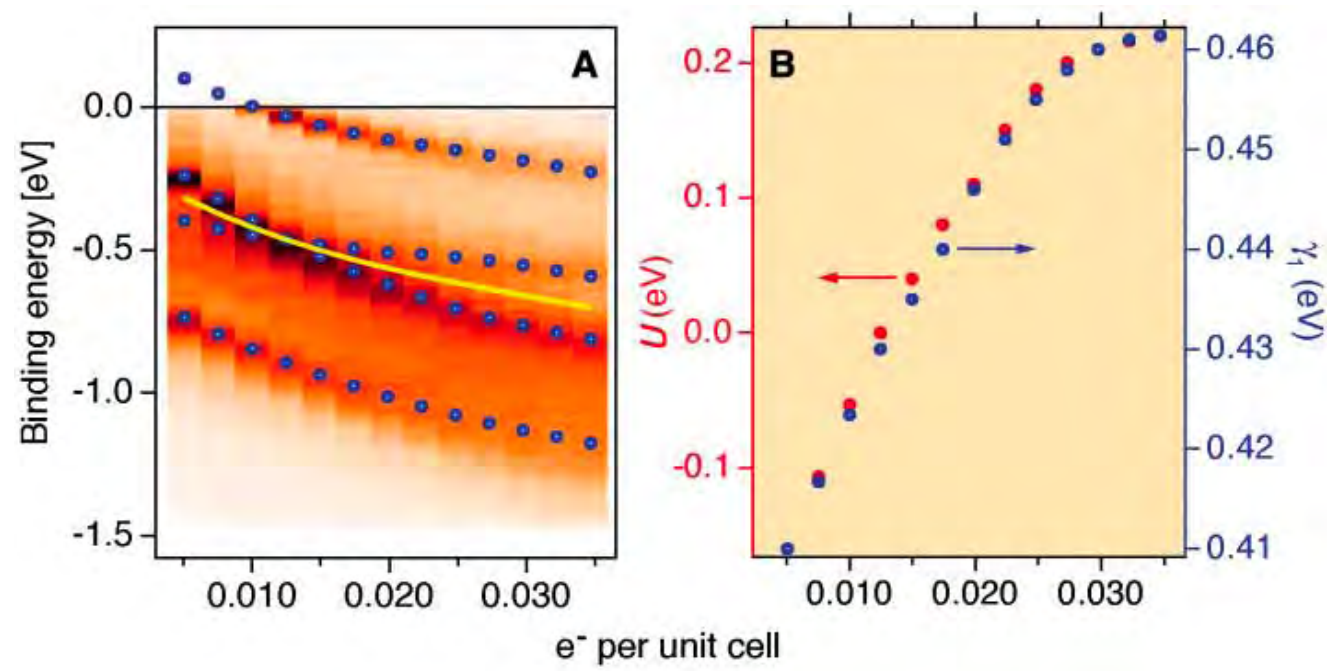

Figure 13: Variation of states at the K point with increasing potassium coverage. (A) The image map shows the energy distribution curve at $K$ as a function of potassium coverage. The blue markers are the fitted positions of the tight-binding $\pi$ and $\pi^{*}$ bands, and the yellow line indicates $E_{D}$. The closing and reopening of the gap between $\pi$ and $\pi^{*}$ states are clearly shown. (B) The influence of doping concentration on the band parameters $U$ and $\gamma_{1}$. From Ohta et al. [52].

conduction bands can thus be manipulated. We have chosen a chemical way as a means of achieving this, i.e. potassium doping, but the switching functionality may be readily induced 


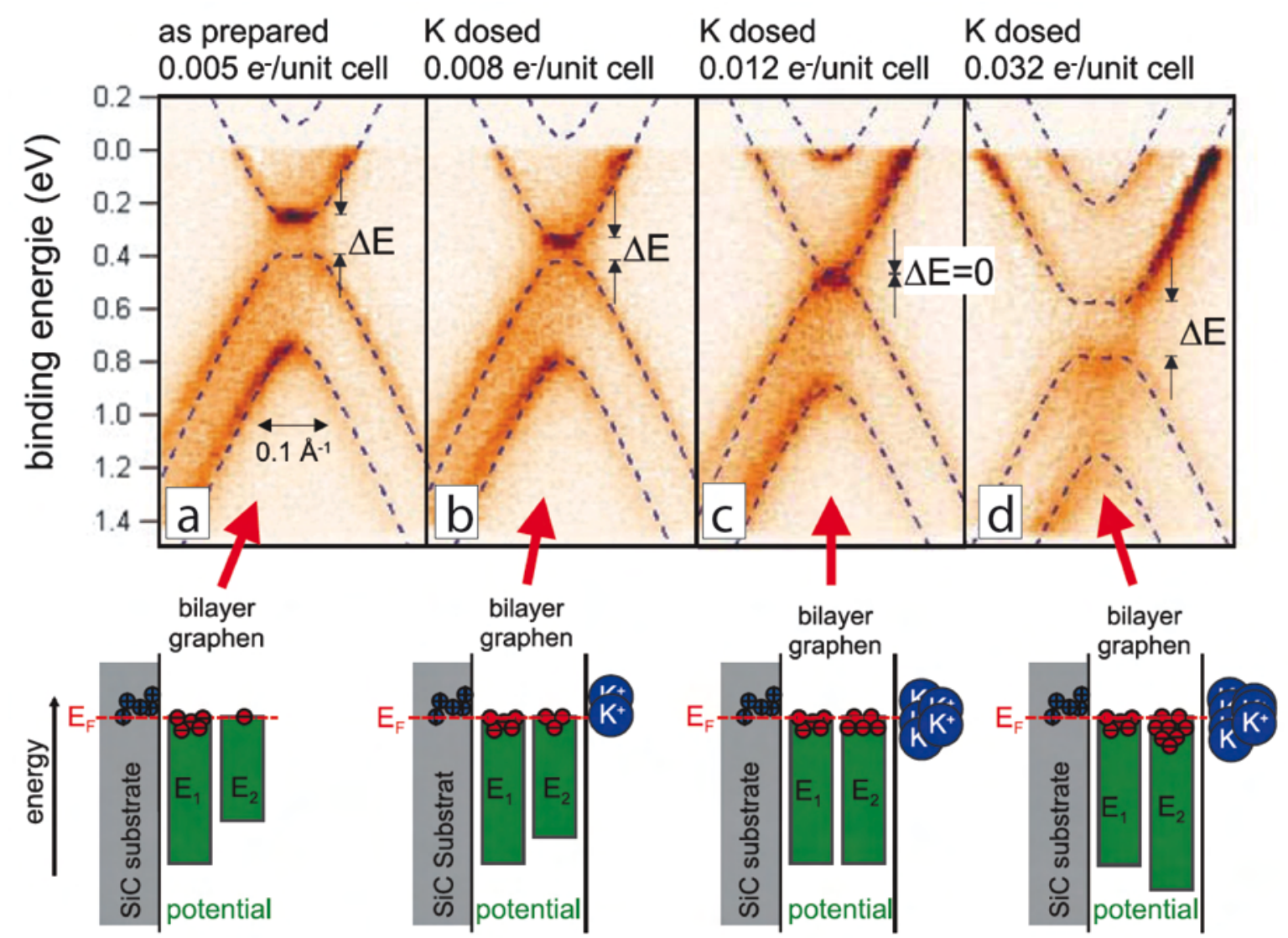

Figure 12: Closing and opening of the band gap in bilayer graphene induced by potassium adsorption. The left panel shows the as-prepared bilayer on SiC(0001). Charge transfer into the bottom layer gives rise to different on-site Coulomb potentials $E_{1}>E_{2}$ which results in a band gap. Doping of the top layer by potassium adsorption reduces the difference between the onsite potentials. If $E_{1}=E_{2}$ the gap is closed. Further $K$ dosing increases the potential difference again, which reopens the gap (see also schematic drawing in Figure 10. The increasing shift of $E_{D}$ below $E_{F}$ is due to the increasing occupation of the $\pi^{*}$-bands. From Seyller et al. [47].

by an electric field across the bilayer in a device structure, in such a way that the potentials on either layer have opposite sign [21]. Note, however, that for a switching functionality to work in a transport device, the gap opening needs to be located at or near the Fermi level; it appears that this has now been achieved in a double gate device configuration [117].

The adsorption of alkali metal atoms is an efficient process to induce (enhanced) n-type doping into the graphene layers; however, it is desirable to be able to induce p-type doping also, for example in order to prepare graphene films on silicon carbide in which the Fermi level coincides with the Dirac point, or to prepare (as yet hypothetical) 2D laterally 
inhomogeneous structures, in which regions of n- and p-type vary. P-type doping has recently been achieved by the deposition of sub-monolayer quantities of bismuth, antimony and gold [118], and through adsorption of $\mathrm{NO}_{2}[119,120]$; it has also been studied theoretically, and experimentally using transport measurements in graphene flakes [121], and the chemically induced change of contact potential has been applied to use graphene as a most sensitive chemical sensor [122]. Consider the set of photoemission images from a graphene layer prepared on $\mathrm{SiC}(0001)$ in Figure 14. The clean surface exhibits the Dirac crossing point of the $\pi$ bands at about $0.45 \mathrm{eV}$ below $\mathrm{E}_{\mathrm{F}}$, i.e. the normal $\mathrm{n}$-type doping found in this system; this doping was first observed by Rollings [64], and has also been predicted theoretically $[68,123]$. Depositing 0.28 monolayers of bismuth per graphene unit cell moves the Dirac point up by about $0.1 \mathrm{eV}$, and with subsequent depositions a situation is reached (Figure $14 \mathrm{c}$ ) where it is located about $0.2 \mathrm{eV}$ below $\mathrm{E}_{\mathrm{F}}$. While bismuth and antimony are only able to compensate the substrate-induced n-type doping to some extent, gold can drive the Dirac point to about 100 meV above $\mathrm{E}_{\mathrm{F}}$, i.e. into the real p-type doping as shown in Figure $14 \mathrm{~d}$. Upon $\mathrm{NO}_{2}$ doping, the Fermi energy can be shifted even more strongly, by as much as $0.8 \mathrm{eV}$, and the charge carriers can be tuned in a wide range from $0.7 \times 10^{13} \mathrm{~cm}^{2}$ electrons to $1.2 \times 10^{13} \mathrm{~cm}^{2}$ holes; moreover, this change is reversible when $\mathrm{NO}_{2}$ is desorbed either through the impact of high energy photons, or high temperature annealing to desorb the $\mathrm{NO}_{2}$ molecules. The Fermi velocity is found to be nearly constant for all doping levels to within $20 \%$ of the initial value [120].

\section{The band structure of single layer graphene: many-body effects}

So far, we have restricted the discussion of the electronic structure of single and few layer graphene to a single particle interpretation of the band structure in terms of tight-binding bands, for example. This view neglects many body effects which play a role, to a larger or lesser degree, in a real solid. With the recent discovery of superconductivity in carbon 
nanotubes [124], alkaline-metal-doped $\mathrm{C}_{60}$ crystals [125] and graphite intercalation compounds [126] with relatively high transition temperatures, there is a strong interest in the influence of many-body interactions on the electron dynamics in carbon-based systems. Graphene, as the building block for all of these materials, is therefore a model system for this entire family also in this respect. These interactions are especially interesting owing to the effectively massless, relativistic nature of the charge carriers following from the formal equivalence of the Schrödinger wave equation to the relativistic Dirac equation for graphene as discussed in detail in the preceding sections. Angle-resolved photoemission is particularly well suited to the study the details of many-body interactions in solids, since it gives access not only to the group velocity and Fermi surface, but also to the constant-energy surfaces for all occupied states and the full occupied band structure $\mathrm{E}(\mathrm{k})$. Recent advances in spectral resolution and the ability to map the distribution of photoemission intensities in energy and momentum space have led to the development of angle resolved photoemission as a tool of central importance in the study of many body processes in solids. Soon after the technical development of imaging detectors, it was shown that the scattering rate in solids can be extracted from the so-called momentum distribution curves (MDC), i.e. angle-resolved photoemission intensity plotted as a function of momentum at fixed energy. This technique, proposed by the Brookhaven group [127] is particularly elegant, and much of the modern high resolution photoemission work of self energy effects and mass renormalization have their origins in their groundbreaking study.

The electronic properties of solids are determined by the quantum states of the conduction electrons. Band theory such as used above for the description of the $\pi$ bands in single and few layer graphene traditionally only accounts for their interaction with the static ion lattice. However, coupling to further microscopic degrees of freedom can alter the electron dynamics and even lead to new many-body ground states not foreseen in that picture. A prominent example is the interaction with lattice vibrations (phonons) [128], which enhances the 
effective electron mass on the corresponding energy scale and can lead to conventional superconductivity. Interaction of conduction electrons with elementary excitations leads to a change ("renormalization") of electronic energies, i.e. to deviations of their band dispersion from that expected for the non-interacting case. At low binding energies, the electrons become "dressed" by excitations, thereby forming quasiparticles of increased mass. As illustrated in Fig. 15 a) [129], this is reflected in a reduced slope of the electron band, which is inversely proportional to the electron mass. Beyond the characteristic energy scale $\omega_{0}$ of the coupled excitations, the electrons resume their non-coupling band dispersion. Quasiparticle formation due to interaction with phonons as depicted in Fig. 15b has been reported by angle-resolved photoelectron spectroscopy (ARPES) $[130,131]$ and is intensely investigated (and debated) in the context of the search for the origins of high temperature superconductivity [132] .
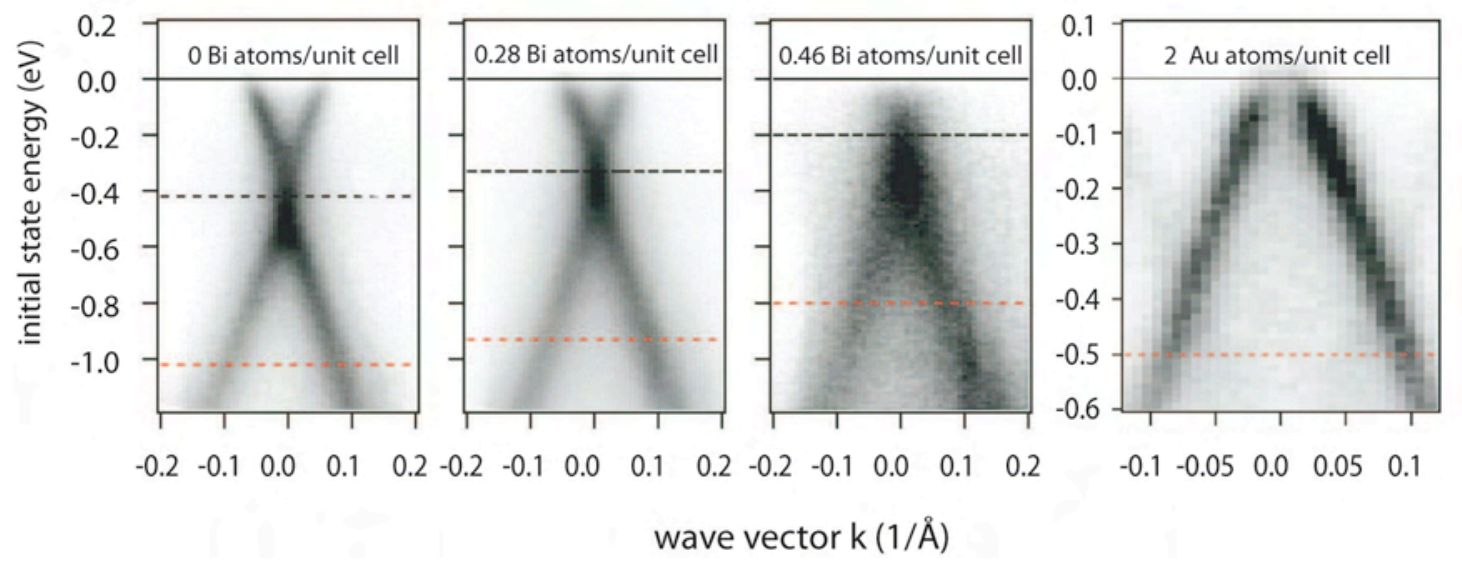

Figure 14. Experimental band structure of single layer epitaxial graphene doped with bismuth and gold atoms. (a) pristine graphene layer, $b, c$ ) increasing amounts of bismuth atoms. d) $p$ type doping induced by a layer of gold with a coverage of two atoms per unit cell of graphene. From Gierz et al. [118]. 

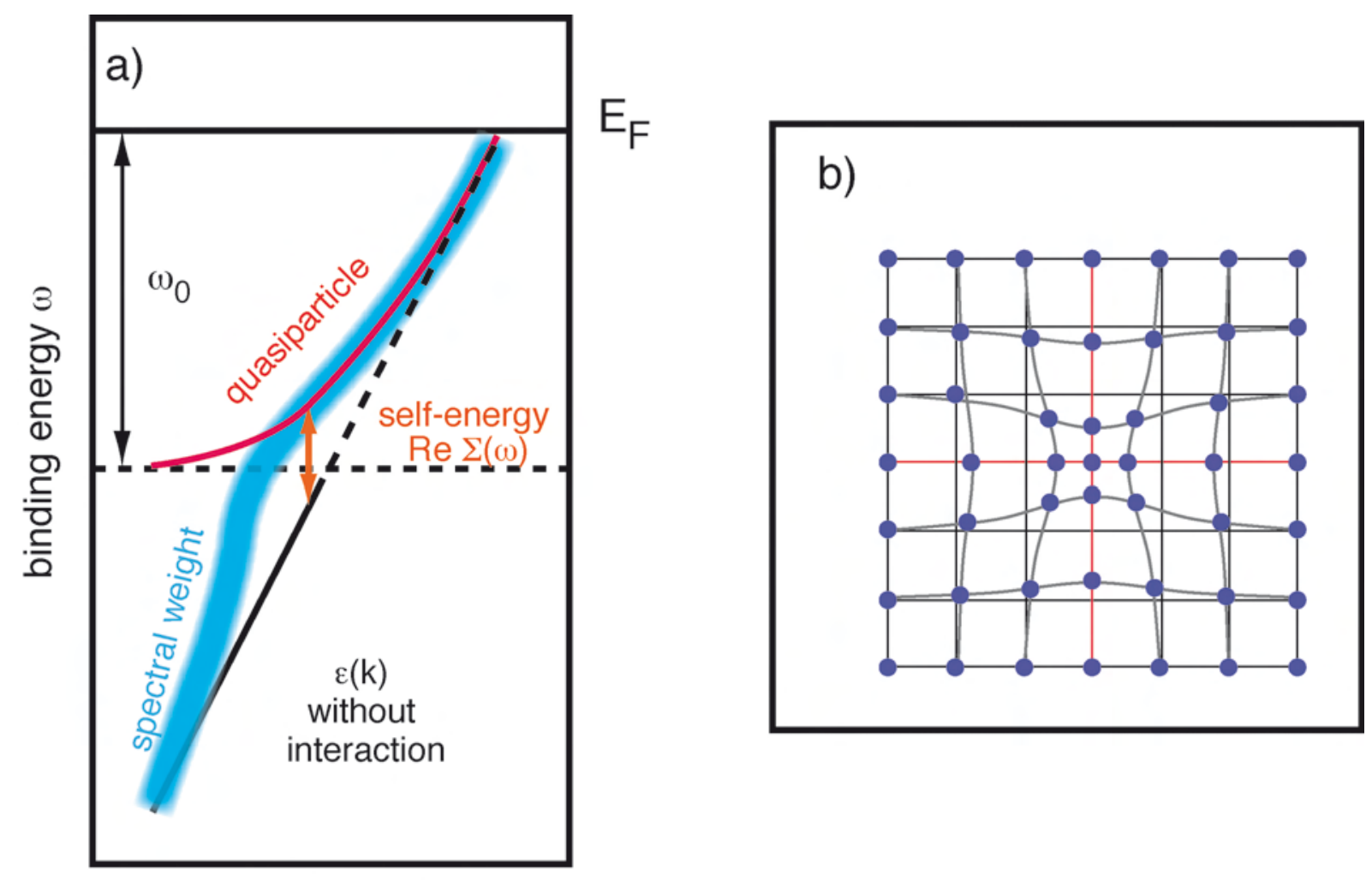

crystal momentum

Figure 15: Schematic display of quasiparticle formation. a )The electron is dressed with the excitation up to an energy $\omega_{0}$ below the Fermi energy, leading to a mass enhancement. b) Electron-phonon coupling implies a distortion of the crystal lattice surrounding the electron. From Schaefer et al., [128]

Under conditions of sufficient energy and momentum resolution, the experimentally determined spectral width of the photoemission contours can be taken to be the inverse of the mean free path, and the experimentally determined $\mathrm{E}(\mathrm{k})$ is taken as a measure of the manybody spectral function $\mathrm{A}(\mathrm{k}, \omega)$. This spectral function is in turn related to the electronic selfenergy $\Sigma(\mathrm{k}, \mathrm{w})$ through

$$
A(k, \omega)=\frac{|\operatorname{Im} \Sigma(k, \omega)|}{\left(\omega-\omega_{b}(k)-\operatorname{Re} \Sigma(k, \omega)\right)^{2}+(\operatorname{Im} \Sigma(k, w))^{2}}
$$

(see Ref. [133] and references therein). Here $\omega$ is the measured binding energy and $\omega_{b}(k)$ is the "bare band" dispersion defined below (in eq. $(11), \hbar=1)$. Here the approximation is made that $\Sigma(\mathrm{k}, \omega)$ is $\mathrm{k}$-independent. In this form, one can show that $\mathrm{A}(\mathrm{k}, \omega)$, when evaluated at constant energy $\omega$, is a Lorentzian function whose width is given by $\operatorname{Im} \Sigma$ representing the 
inverse lifetime (proportional to the inverse mean free path). Equation 11 is valid when the scattering rate of the charge carriers (expressed in energy units) is not too large compared to their energy. The charge carriers are then referred to as quasiparticles (QPs); in our measurements, the QPs are holes which have been injected as part of the photoemission process.

One can draw an analogy between QPs propagating in a scattering medium and light traveling in a lossy optical medium [32]. Such a medium is characterized by a complex dielectric function, and the effects on the light propagation are not only through its absorption but also a dispersion. To satisfy causality, the real and imaginary parts of the dielectric function are related by a Kramers-Kronig (Hilbert) transformation. Similarly, the propagation of QPs in a scattering medium leads not only to inelastic scattering (whose lifetime is contained in the imaginary part of $\Sigma$ ) but also renormalization of the carrier's energy, contained in the real part of $\Sigma(\mathrm{k}, \omega)$. These real and imaginary parts of $\Sigma(\mathrm{k}, \omega)$ are also related by a Hilbert transform, and the function $\operatorname{Re} \Sigma(\mathrm{k}, \omega)$ is defined as the difference between the measured carrier energy $\omega$ and the "bare" band energy $\omega_{\mathrm{b}}(\mathrm{k})$ (that is, in the absence of scattering interactions), as indicated in Eq. 11.

Following this formalism, ARPES can determine the energy-dependent lifetime due to scattering from other excitations in the system [134]. Moreover, the coupling among quasiparticles can be evaluated, a process which is fundamental towards understanding superconductivity, for example.

The analysis focuses in particular on the interaction of the carriers with-electron-hole pair excitations within the Fermi-liquid model, and with plasmons. Departure of the electron dynamics from Fermi liquid behavior in graphite has already been attributed to the special shape of the graphene band structure $[97,135,136]$, whereas electron-plasmon scattering has 
(a) $1 \mathrm{ML}$, as grown
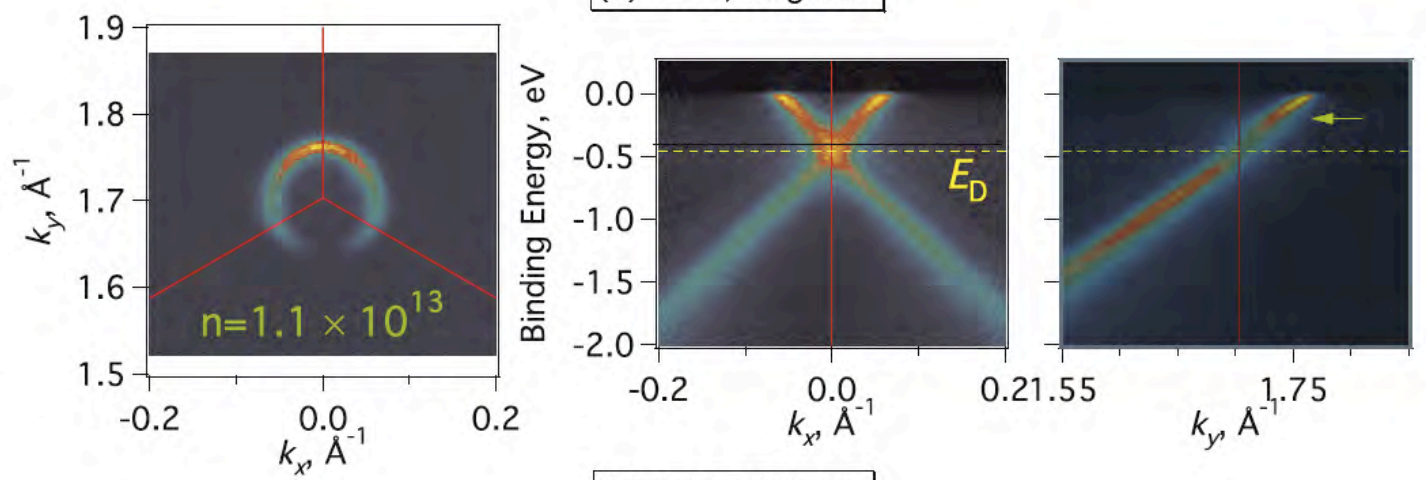

(b) $1 \mathrm{ML}, \mathrm{K}$-dosed
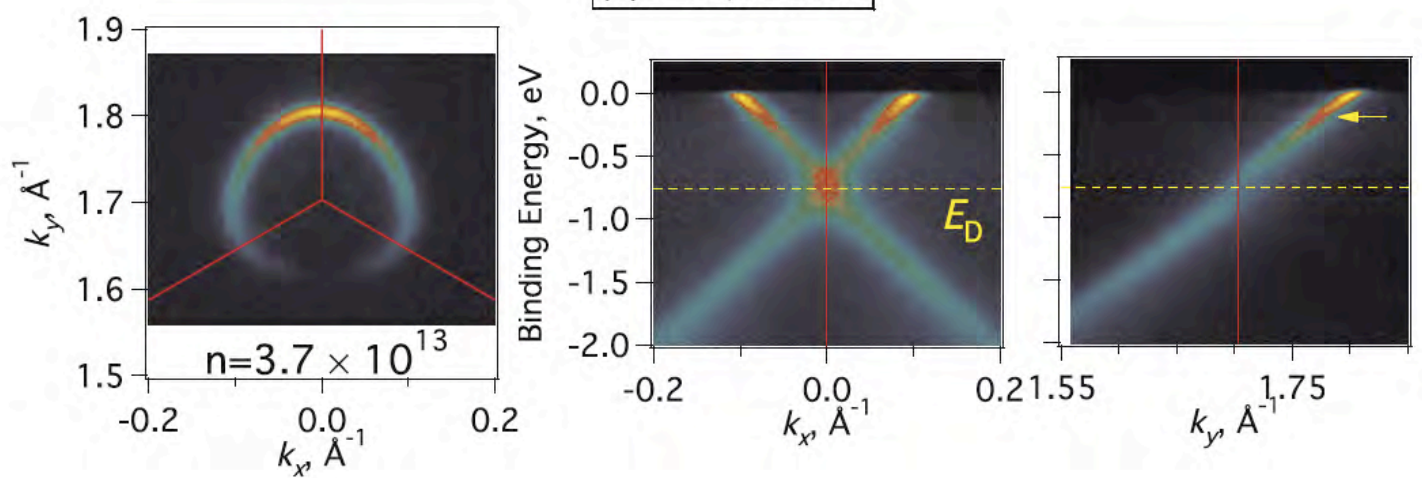

Figure 16. Experimental Fermi surfaces (left) and band structures (middle, right) for (a) asprepared monolayer graphene and (b) graphene dosed with $K$ atoms. The middle and right panels are taken along orthogonal directions through the $K$ point as indicated, i.e. along $k_{x}$ at constant $k_{y}=0$ and vice versa. The doping levels in electrons per $\mathrm{cm}^{2}$ are indicated. The phonon kinks at $200 \mathrm{meV}$ are indicated by arrows. From Bostwick et al., [32].

been proposed as a key coupling process in superconductivity in general [137], and of the cuprates in particular [138]. Angle-resolved photoemission spectroscopy (ARPES) can probe the scattering rate at different energy scales, and therefore can access these many-body couplings directly [132]. For undoped graphene, the Fermi energy $E_{F}$ lies at the Dirac point $E_{D}$, and many of the interesting properties of graphene arise from the fact that the band crossing at $E_{D}$ has no gap, a fact that is amply demonstrated in transport experiments $[1,110]$. Upon doping the graphene by either deposition of foreign atoms [41,52,53], molecules [120], [121] or in a gated geometry $[1,21,110]$, the carrier density can be easily manipulated. With this control, one can systematically study the many-body interactions in graphene as a function of doping. While transport measurements on doped graphene can determine the 
relevant properties such as group velocity and lifetime of carriers on the Fermi surface, which is defined as the contour $\mathrm{E}(\mathrm{k})$ taken at the Fermi energy, angle-resolved photoemission spectroscopy (ARPES) is a complementary tool giving access to the entire electronic band structure as demonstrated in the previous sections. As shown there, angle resolved photoemission also gives a very precise determination of graphene film thickness, because of the distinct signatures of the single and few layer graphene band structure [54].

The detailed spectral function by ARPES from graphene was reported by Bostwick et al. [53] and showed rich spectral features. As discussed in section 2, the formation of graphene on $\mathrm{SiC}(0001)$ proceeds through a $(6 \sqrt{3} \times 6 \sqrt{3}) \mathrm{R} 30^{\circ}$ reconstruction at the graphite-SiC interface, a non-interacting "0th" graphene layer whose electronic structure resembles graphene only insofar as it has an intact $\sigma$-like bands (derived from $\mathrm{sp}^{2}$-hybridized in-plane bonds) but lacking the $\pi$ bands characteristic of the out-of-plane $\mathrm{p}_{z}$ states of graphene (cf. Figure 4). The presence of such a zeroth layer is important because it saturates the underlying SiC dangling bonds while forming a template for a subsequent first graphene overlayer. From symmetry considerations, it is known that the $\pi$ bands from the latter and the $\sigma$ bands of the former cannot interact. Therefore, the chemical interaction of the first graphene layer with the substrate is very weak, and the $\pi$ bands of graphene on $\mathrm{SiC}$ are to a very good approximation the same as those of free-standing graphene.

As discussed in the preceding sections, the conical band structure $\mathrm{E}(\mathrm{k})=\mathrm{v} \boldsymbol{k}$ near the $\mathrm{K}$ point of the Brillouin zone is directly mapped in photoemission as shown in Figure 6, for example. Let us now examine this region of the bands in more detail, and take a look at the Fermi surface (left diagram of Figure 16 a) [32]. The increase in diameter of the latter as the $\mathrm{n}$-type doping is increased by adsorption of potassium (lower panel) is obvious, and this is a method for estimating the coverage based on the assumption that each $\mathrm{K}$ atom gives up its valence electron. One immediately recognizes the expected nearly linear dispersion as well as 
the Dirac crossing point (middle panels) in the bands at the Dirac energy $E_{D}$. There is also a nontrivial change in band intensity when traversing around the Fermi contour. Because of this effect, when one samples the band structure in the $\mathrm{k}_{\mathrm{y}}$-direction, only one of the two expected bands is observed; the other is extinguished (right panels; see also Figure 6). This asymmetry in constant-energy angular maps of photoemission reflects the chirality of charge carriers in graphene; it does this in a manner that can be evaluated quantitatively to investigate the strength of symmetry-breaking effects produced by the underlying substrate, or by doping in the bilayer [139], a subject that is discussed in more detail at the end of this section.

An important feature of single layer graphene data is the appearance of kinks in the energy band structure below $\mathrm{E}_{\mathrm{F}}$ [53]. These kinks occur at two energy scales. First, a slight kink at $\sim 200 \mathrm{meV}$ below $\mathrm{E}_{\mathrm{F}}$, which is hardly visible on the large energy scale plotted in Figure 16, but is accompanied by pronounced sharpening between $200 \mathrm{meV}$ and $\mathrm{E}_{\mathrm{F}}$, is evident from the data displayed in a false color scale. This kink is similar to ones which have been observed at such an energy scale in graphite $[104,140]$ and bilayer graphene as shown above, and have been attributed to electron-phonon scattering. The occurrence of the kink was discussed within the spectral function formalism in eq. 11 and depicted in Figure 15. There is an observable increase in line width of the band at binding energies greater than $200 \mathrm{meV}$, signifying a decrease in the lifetime of the states as electrons absorb or emit phonons. This interpretation is based on two observations: first, because of the energy scale, which corresponds to the in-plane LO and TO phonons of graphene [53], and second, because the kink feature's energy scale remains constant with doping, as expected for the small doping levels considered here.

Second, it is obvious from the data in the upper panels, where both bands have equal intensity, that the region of the crossing of the bands seems spread out in energy. In the lower panels, where one band is extinguished, it appears that this spread is associated with a second 
kink feature which is at the Dirac crossing point of the bands. Unlike the phonon kink, this anomaly moves to higher binding energy with doping, and must therefore be somehow associated with the Dirac energy ED. It is stronger at higher doping, and is associated with a change in line width - the bands are locally broadened around ED [32].

The deviations from the bare band are sensitive to doping as shown in Fig. 17 b-d and along the orthogonal direction in Fig. $17 \mathrm{f}-\mathrm{h}$. Similar to graphite, doping graphene by K deposition shifts the bands more or less rigidly to higher binding energy. Whereas the energy of the kink at $200 \mathrm{meV}$ does not change, the deeper-energy kink strengthens and follows $\mathrm{E}_{\mathrm{D}}$ with doping, demonstrating that it is associated with electrons with an energy near $E_{D}$. In order to provide an interpretation of the entire spectral region within the range of binding energies from $\mathrm{E}_{\mathrm{F}}$ to about $1 \mathrm{eV}$ below $\mathrm{E}_{\mathrm{F}}$, Bostwick and coworkers [53] analyzed the experimental data within the the k-independent approximation $[133,141]$, where $\operatorname{Im} \Sigma(\omega)$ is
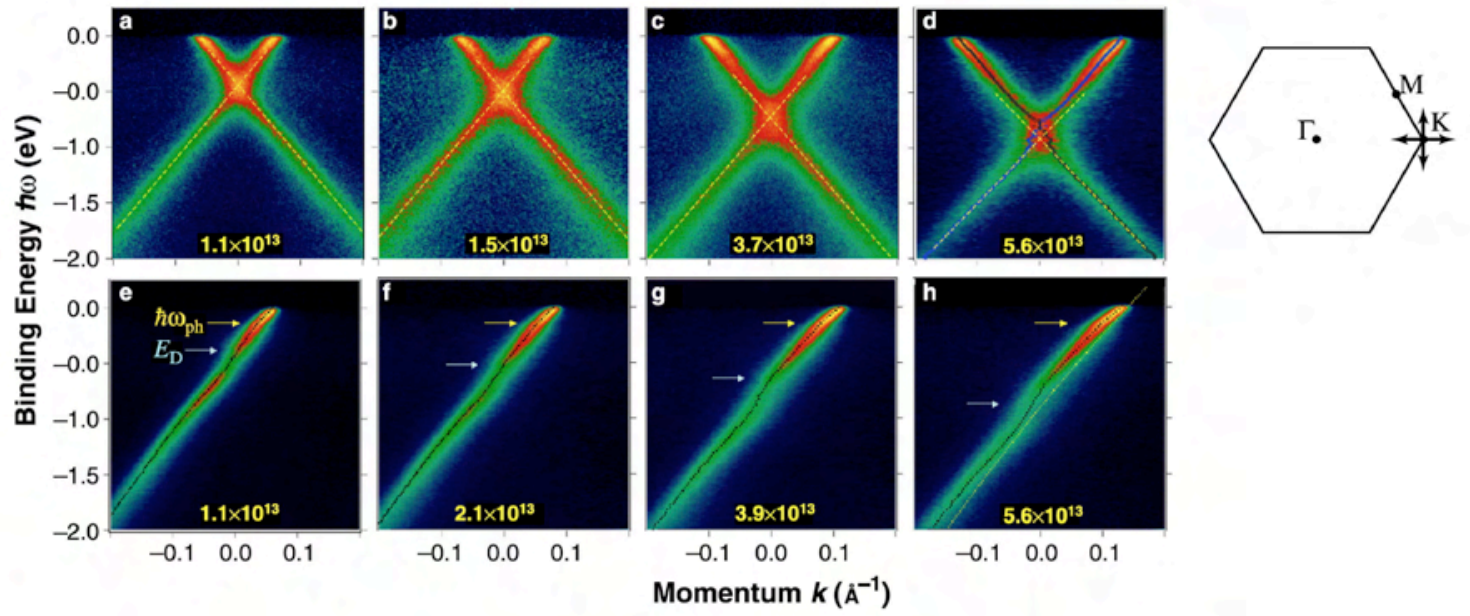

$-0.1 \quad 0.0 \quad 0.1$

Figure 17: The band structure of graphene near the Fermi Level. (a)-(d) Experimental energy bands along the vertical double-arrow in the inset as a function of doping. The dashed lines are an extrapolation of the lower bands (below the Dirac crossing energy $E_{D}$ ). The electron density (charge per $\mathrm{cm}^{2}$ ) is indicated on each panel. (e)-(h) Band maps for similar dopings acquired along the horizontal double arrow in the inset. The non-linear, or "kinked" dispersion of the bands is evident from the fitted band position (dotted lines). From Bostwick et al. [53].

proportional to the Lorentzian line width of the momentum distribution curve (MDC) taken at 
constant energy $\omega \cdot \operatorname{Re} \Sigma(\omega)$ is computed from $\operatorname{Im} \Sigma(\omega)$ through a Hilbert transform, and the full spectral function $\mathrm{A}(\mathrm{k}, \omega)$ is reconstructed using the computed $\operatorname{Re} \Sigma(\omega)$ and compared with experiment. In order to find out whether such a procedure can be carried out selfconsistently, it should first be established that the kinks in the bands and the linewidth variations are consistent with each other. Figure $17 \mathrm{~d}$ ) and h) and Figure 18 a) show an experimentally acquired spectral function $\mathrm{A}(\mathrm{k}, \omega)$ for relatively highly doped graphene $(\mathrm{n}=$ $\left.5.6 \times 10^{13} \mathrm{~cm}^{-2}\right)$. The band positions $\omega=\omega_{\mathrm{b}}(\mathrm{k})+\operatorname{Re}(\mathrm{k}, \omega)$ are determined by fitting MDCs (i.e. individual constant-energy slices) to Lorentzian functions. The positions are plotted in Figure 18 a) (black line).

In order to converge to a self-consistent interpretation, the authors adopt the following procedure: a second-order polynomial is taken as a trial bare band $\omega_{b}(k)$. Given this $\omega_{b}(k)$, the MDC widths (units of $\AA^{-1}$ ) are transformed into the function $\operatorname{Im} \Sigma(\mathrm{k}, \omega)$ (units of eV). This function is smoothed and then Hilbert transformed into a trial $\operatorname{Re} \Sigma(\mathrm{k}, \omega)$ function. $\operatorname{Re}(\mathrm{k}, \omega)$ can also be obtained by subtracting the trial bare band from the fitted band position. These two $\operatorname{Re} \Sigma(\mathrm{k}, \omega)$ functions can then be compared and the trial bare band adjusted until the models $\operatorname{Re} \Sigma(\mathrm{k}, \omega)$ and $\operatorname{Im} \Sigma(\mathrm{k}, \omega)$ are in good agreement with the experimentally extracted curves; for details see [32,53]. As a check of self-consistency, a trial spectral function $A(k, \omega)$ can be generated from only the fitted MDC widths and the mathematically transformed $\operatorname{Re} \Sigma$ $(\mathrm{k}, \omega)$, shown in figure $18 \mathrm{~b})$. It is in excellent agreement with the experimentally determined function in Figure 18 a).

This self-consistency gives confidence that all kinks in the bands originate from manybody interactions as contained within the spectral function, and not from details of the single particle band structure. The observed kink structure must therefore be assigned to an energydependence of the observed scattering rate, which is proportional to the measured MDC line widths. To interpret these data, the authors consider three processes: decay of the carriers by 
electron-phonon (e-ph) coupling, by electron-hole $(\mathrm{e}-\mathrm{h})$ pair generation and by emission of collective charge excitations (plasmons) through electron-plasmon (e-pl) coupling. The experimental scattering rate and its contribution to $\operatorname{Im} \Sigma(\mathrm{k}, \omega)$, determined from the width of the peak in the momentum distribution curve, is shown as the red dots in Figure 19 d. From the shape of the experimental scattering rate curve, there are four regions of interest I - IV: (I) the phonon energy scale $\omega_{\mathrm{ph}}<\omega<0$, (II) the Dirac energy scale $\omega_{\mathrm{D}}<\omega<0$, (III) $2 \omega_{\mathrm{D}}<\omega$ $<\omega \mathrm{D}$, and (IV) $\omega<2 \omega \mathrm{D}$.

First, the kink near $\mathrm{E}_{\mathrm{F}}$ (region I) is attributed to electron-phonon coupling as described previously for metals $[127,130,142]$, and for bulk graphite [104]. In this process, photoholes decay by phonon emission (see also Figure 15). From graphite's phonon density of states [143], the e-ph contribution to the scattering rate and thus $\operatorname{Im} \Sigma$,(green curve in Figure $19 \mathrm{~d}$ ) is calculated [128] and an e-ph coupling constant $\lambda \approx 0.3$ is found. Although this is a factor of five larger than predicted [144] for $\mathrm{n}=5.6 \times 10^{13} \mathrm{~cm}^{-2}$, comparison with the experimental data shows that this provides an accurate description of $\operatorname{Im} \Sigma$ in region I (for details see [53]). The observed increase of the kink's strength with n (see Fig. $17 \mathrm{e}-\mathrm{h}$ ) is expected from the increase in the size of the Fermi surface, although the $200 \mathrm{meV}$ energy scale remains constant because the $\mathrm{K}$ atoms are not likely to alter the phonon band structure at this energy.

Consider now the decay of the photohole by excitation of an electron from below to above $\mathrm{E}_{\mathrm{F}}$, thereby creating an e-h pair. In Landau's Fermi-liquid theory, the scattering rate from such processes increases proportional to $\omega^{2}$ away from $\omega=0$, reflecting the growing number of possible excitations that satisfy momentum and energy conservation. However, the linear dispersion of the graphene bands and the presence of the Dirac crossing below $\mathrm{E}_{\mathrm{F}}$ drastically modify this picture [145]. A hole just above $E_{D}$ can easily decay through many possible $\mathrm{e}-\mathrm{h}$ creation events, for example, as shown schematically in Fig. 19 b), and an $\omega^{\alpha}(\alpha \sim 1.5)$ dependence of $\operatorname{Im} \Sigma(\mathrm{k}, \omega)$ is found in regions I-II. In Fermi-liquid theory for 3D solids, $\alpha=2$ 
is derived, and for a $2 \mathrm{D}$ solid where a dependence $\omega^{2} \ln \omega$ was predicted $[146,147]$. The influence of the unique band structure on quasiparticle scattering rates in graphene was analyzed by several groups $[135,148-151]$. The situation is quite different from a normal 2D

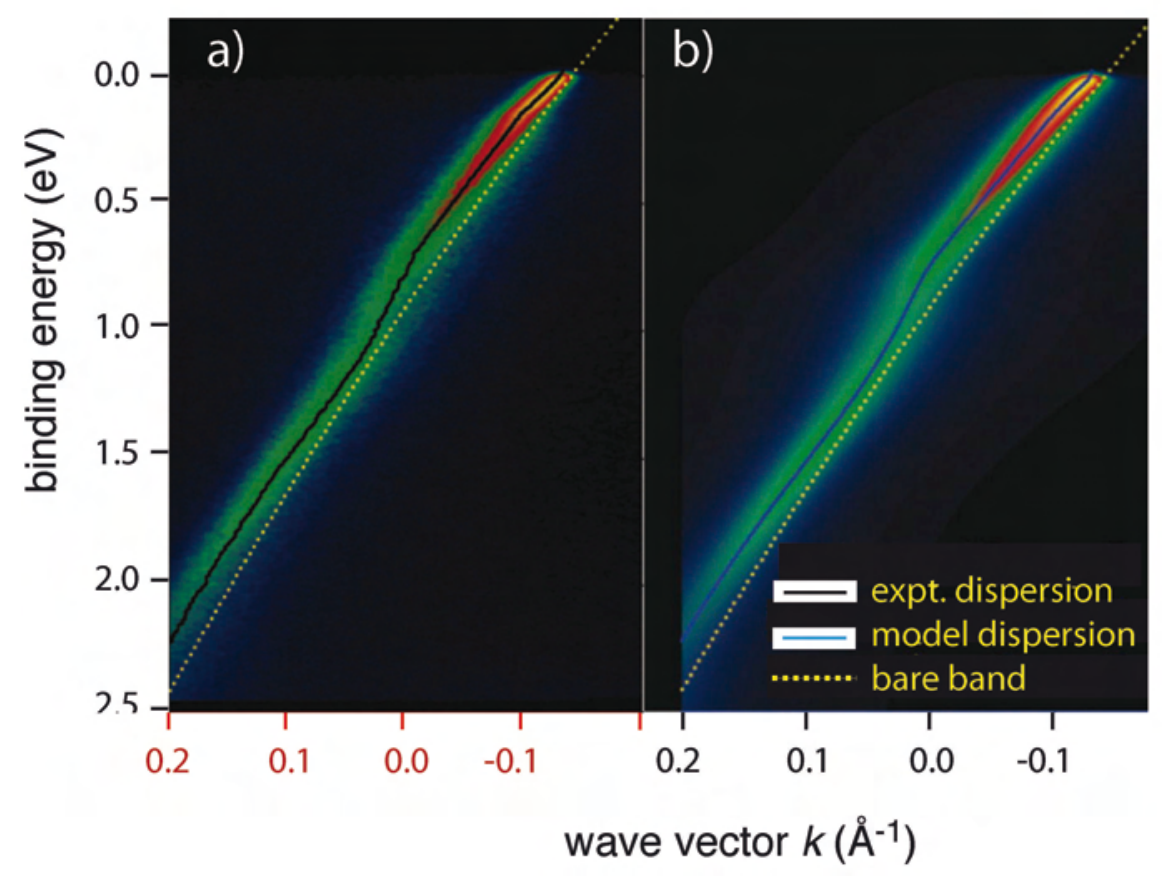

Figure 18. Spectral function of doped graphene. (a) The experimentally determined spectral function for graphene doped with $K$ atoms (total doping $n=5.6 \times 10^{13} \mathrm{~cm}^{-2}$ ). The solid line is the fitted band position $\omega_{b}(k)+\operatorname{Re} \Sigma(k, \omega)$, the dotted line is the bare band $\omega b(k)$. (b) A model spectral function generated using only the measured $\operatorname{Im}(k, \omega)$ and the bare band $\omega_{b}(k)$. Adapted from Bostwick et al. [53].

electron gas since a hole originating at $\omega$ just below $E_{D}$ has few possible decays with sufficient momentum transfer to excite an e-h pair [Fig. 18c]. This causes a sharp reduction in the scattering rate in region III, seen in the red curve in Fig. 19 d). Only for energies $\omega$ larger than approximately $2 \omega_{D}$, region IV, does e-h pair generation become favorable again. The electron-electron and electron-phonon processes can explain the observed MDC widths in regions I, II and IV. In region III, however, decay by e-h pair creation is virtually not allowed, yet the observed scattering rate has a peak rather than a dip (experimental data in Fig. 19). 
This peak may be explained by decay through plasmon emission, i.e. oscillations of the

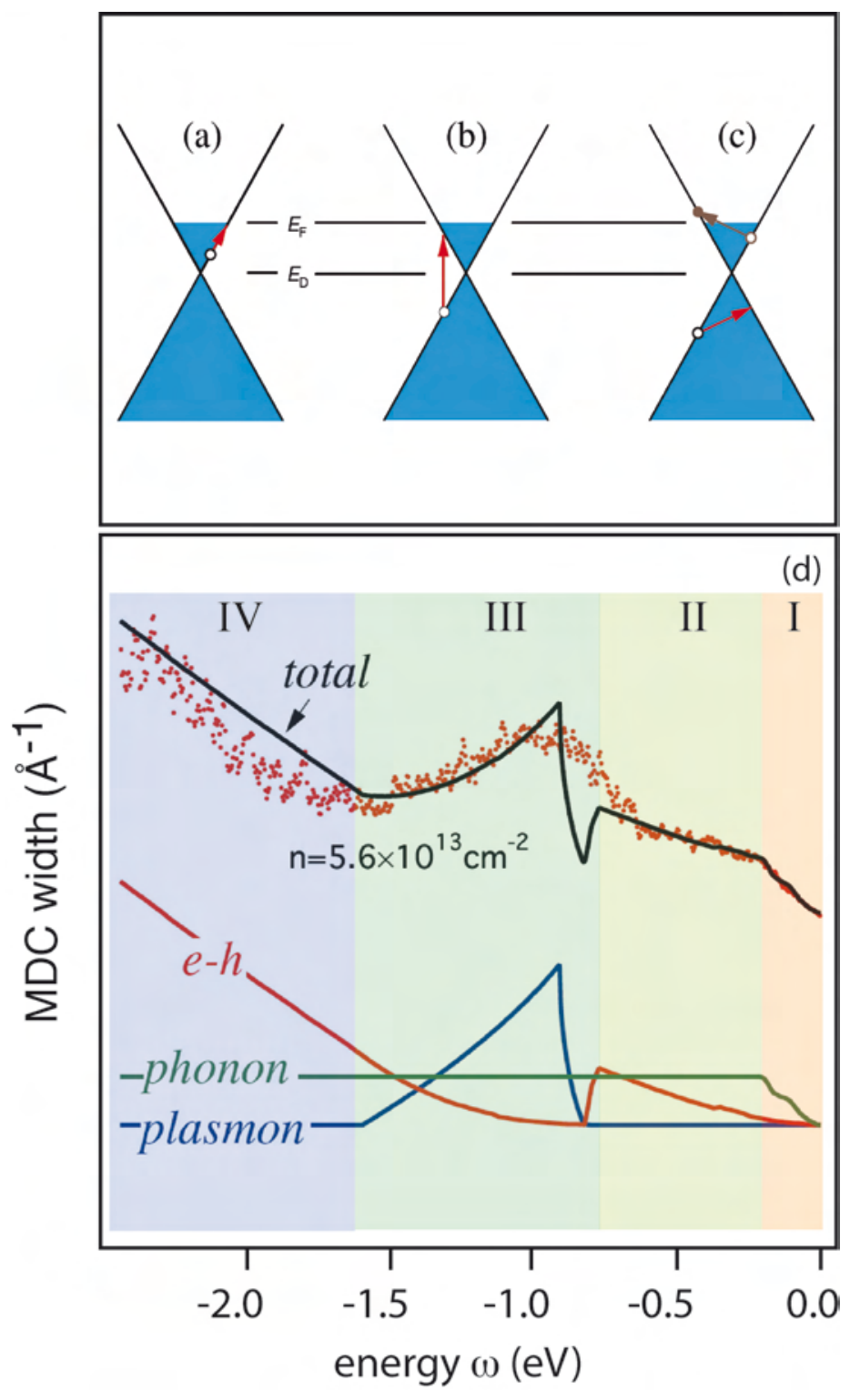

Figure 19: Possible many-body decay process in n-doped graphene. (a) decay by electronphonon emission (b) decay by electron-plasmon emission (c) decay by electron-hole pair generation. d) Measured spectral linewidth of peaks in MDCs for graphene (red dots), derived by performing a line shape analysis for each binding energy. The simulated total scattering contribution (black line) and the partial contributions due to decay into phonons (green), electron-hole pairs (red) and plasmons (blue) are calculated for the specific doping. These interactions contribute differently in regions I-IV defined as follows: (I) the phonon energy scale $\omega_{p h}<\omega<0$, (II) the Dirac energy scale $\omega_{D}<\omega<0$, (III) $2 \omega_{D}<\omega<\omega_{D}$, (IV) $\omega<$ $2 \omega_{D}$. Adapted from Bostwick et al. [53].

electron gas. 
In graphene, the charge carriers near the $\mathrm{K}$ point have zero effective mass and travel like photons at constant speed $\mathrm{c} *$, but unlike photons they have charge and are therefore subject to collective oscillations such as plasmons. Although a full treatment of the electron-plasmon interaction is difficult near the Dirac point, a simple model suffices to explain how electronplasmon coupling can enhance the scattering rate below $\mathrm{E}_{\mathrm{D}}$. Two-dimensional plasmons have a dispersion relationship $\omega_{\mathrm{pl}}(\mathrm{q})=4 \pi n e 2 \mathrm{q} / \mathrm{m}(1+\varepsilon)$

where $\mathrm{q}$ is the plasmon momentum, $\mathrm{e}$ is the electron charge, $\mathrm{m}$ is the effective carrier mass and $\varepsilon$ is the dielectric constant. For graphene, the rest mass $m_{0}$ is zero near $E_{D}$, but the 'relativistic mass' $\mathrm{m}_{\mathrm{r}}=\mathrm{E} / \mathrm{c}^{2}$ depends on the doping, reaching a maximum of only $10 \%$ of the free-electron rest mass for our samples; this has the notable effect of increasing the plasmon energy $\omega_{\mathrm{pl}}(\mathrm{q})[53]$. Collective plasmon excitations are not really independent of the $\mathrm{e}-\mathrm{h}$ pair excitations, and therefore decay by plasmon scattering is a valid description only outside the range of kinematically allowed e-h processes, which only occurs for decay processes with $\omega$ $\sim \mathrm{E}_{\mathrm{D}}$ and $\mathrm{q} \sim 0$ (as in Fig. 19b), when the plasmon spectrum does not overlap the continuum of e-h excitations. This means that plasmons may have a large effect on the self-energy around $\omega \sim \omega_{\mathrm{D}}$. Using the plasmon dispersion relation, the possible plasmon decays as a function of $\omega$ (Fig. $19 \mathrm{~d}$, blue curve), which is proportional to the scattering rate, can be calculated. This leads to a peak in $\operatorname{Im} \Sigma$ located just below $E_{D}$, whose width and intensity scales with $E_{D}$, as clearly observed in the experimental data. The three principal decay processes (e-h, e-pl, and e-ph) contribute differently to the lifetime in regions I-IV as identified in the traces in the upper part of Fig. $19 \mathrm{~d}$, calculated for a sample with $\mathrm{n}=5.6 \mathrm{x}$ $10^{13} \mathrm{~cm}^{-2}$, and compares favorably with the experimental MDC width; the predicted dip at $E_{D}$ is an artefact of the simplicity of the model, which does not consider interactions between the plasmons and the Fermi-liquid excitations. Considering the influence of the three principal 
decay processes, i.e. electron-hole excitations, electron-plasmon and electron-phonon scattering thus gives a consistent explanation of the shape of the dispersion curve and the trends in the experimental scattering rates. Plasmons have been identified in scanning tunneling spectroscopy of graphene [143], and in a recent first-principles study of quasiparticle line widths, Park and coworkers have considered the influence of these processes and found good agreement between their theoretical results [152] and the experimental data discussed above.

The analysis of the scattering rate near the Dirac point in terms of electron-plasmon scattering by Bostwick et al. has recently been put in question by Zhou et al [153], who proposed that the observed spreading of the bands around $\mathrm{E}_{\mathrm{D}}$ is associated with substrate dependent energy gap at $E_{D}$. Such a gap would be interesting because it suggests an electronic or chemical control of the electronic character (2D semimetal vs. semiconductor) and is proposed on the basis of possible symmetry breaking. A physical interpretation of this gap is the symmetry breaking of the A and B atoms. This occurs for the replacement of C atoms with $\mathrm{B}$ and $\mathrm{N}$ in hexagonal boron nitride, for example, creating a gap of about $5 \mathrm{eV}$. It also occurs in a scenario where the bonding of $\mathrm{A}$ and $\mathrm{B}$ atoms to the zeroth layer is asymmetric. Zhou and coworkers have recently interpreted their angle-resolved photoemission data from monolayer graphene in terms of the opening of a gap between the lower and upper part of the $\pi$ bands, i.e. below and above the Dirac point [153]; they assert that chemical bonds between the graphene film and the substrate break the 'A-B' symmetry of the graphene lattice. As discussed by Bostwick et al. [32] and Rotenberg et al. [154], there are several reasons why the presence of a gap appears most unlikely. First, the interaction between the 1st and 0th layer is very weak, as established by photoemission [55], theory $[155,156]$, and STM [68]. Moreover, the doping dependence shows a clear increase in the spread of the states at the Dirac crossing. If this spread were due to a gap from coupling to the substrate, the coupling strength should be independent of the doping density (or become smaller due to enhanced screening). Also, 
the bands above and below $\mathrm{E}_{\mathrm{D}}$ are misaligned [52]. This misalignment does not occur in the energy gap scenario, but comes naturally when many-body interactions are present. The energy distribution curve (EDC) at the Dirac crossing shows only a single peak, not a split peak as expected in a gap scenario (see Fig. 5 in ref. [32]).

As an alternative explanation for the different interpretations of the spectral data by Zhou et al., and the data presented above, Rotenberg et al. [154] have recently compared spectra of a complete monolayer with those of submonolayer coverages, where small islands of graphene are known to exist from STM and LEEM data [81]. They relate the findings of Zhou et al. to the presence of defects and islands of graphene readily prepared by underannealing. Such films display not only the split or 'gapped' EDC spectrum at K., but also a significant broadening and an elevated diffuse background in the Fermi-level MDCs. Other experimental observations also suggest that such samples are islanded (see Figure 1 in ref [154]). This is also supported by electron microscopy showing an inhomogeneous distribution of small irregular graphene islands, features which can easily explain the data of Zhou and coworkers. The intensity distribution along the Fermi surface such as shown in Figure 20, where photoemission intensity on one side of the Fermi contours is very weak or absent, provides a more stringent test for A-B atom symmetry breaking. The Hamiltonian of one layer of graphene near the K point of the Brillouin zone can be approximated $[12,109,157,158]$ by

$$
H=\left(\begin{array}{cc}
E_{1}+\Delta / 2 & v\left(k_{x}-i k_{y}\right) \\
v\left(k_{x}+i k_{y}\right) & E_{1}-\Delta / 2
\end{array}\right)
$$

where the wave functions $\Psi=\left(\psi_{\mathrm{A}}, \psi_{\mathrm{B}}\right)$ are written in terms of $\mathrm{p}_{\mathrm{z}}$ orbitals centred on the $\mathrm{A}$ and $\mathrm{B}$ atoms in the graphene basis set. The parameter $\Delta$ represents a possible asymmetry between the $\mathrm{A}$ and $\mathrm{B}$ sites. For ordinary graphene, $\Delta=0$ since the atoms are indistinguishable. The off-diagonal terms represent the hopping between A and B sublattices, and $\mathrm{v}$ is the band velocity around ED. The Hamiltonian in equation (13) leads to the by now well-known conical 
band structure $\mathrm{E}(\mathbf{k})=\mathrm{vk}$ when $\Delta=0$, where $\mathbf{k}$ is the momentum relative to one of the $\mathrm{K}$ points at the corner of the graphene Brillouin zone (see Figure 1).

In the symmetric case $\Delta=0$, the intensity on one side of the Fermi contour is strictly zero. Rather than a simple vanishing photoemission matrix element, the cancellation results from the interference between emission from A and B sites. This cancellation, like the Dirac nature of the quasiparticles, and the lack of backscattering, follow from the A-B atom symmetry. If the A-B atom symmetry is broken, not only is a gap opened at $\mathrm{E}_{\mathrm{D}}$ (thereby destroying the massless character), but also the phase cancellation affecting the Fermi surface intensity is destroyed [92]. The magnitude of this effect is illustrated by the polar maps of the measured angular distribution of the band intensity taken about the $\mathrm{K}$ point for monolayer and bilayer graphene (closed and open circles, respectively) in Figure 20. These data were obtained by fitting the momentum distribution curves taken along radial cuts for an energy window $\sim 75$ $\mathrm{meV}$ below $E_{\mathrm{F}}$. For the monolayer, the band intensity is zero within a very low noise floor (about $0.15 \%$ as indicated by the central yellow circle). Shirley derived a simple formula for the symmetric case $\Delta=0$ for monolayer graphene; an extension of this model is shown by the red lines in Figure 20, where the expected angular distributions for a $\Delta=0.0,0.1$ and $0.2 \mathrm{eV}$ (leading to energy gaps at ED of the same values) is shown by the solid red lines. From a comparison of the intensity minimum (black data point) with the experimental noise floor $(<0.015 \%)$ one can conservatively estimate the maximum gap at $\mathrm{E}_{\mathrm{D}}$ to be under $60 \mathrm{meV}$. Since the apparent kink at ED (with a resulting spreading of the states there) is much wider in energy than this, symmetry breaking as being the dominant factor to explain the anomalous dispersion at ED can be ruled out.

\section{Conclusions and outlook}

The "rise of graphene" [4] has taken the scientific world by surprise, and rarely has a new material come along that has both fundamental interest and a wealth of possible practical 

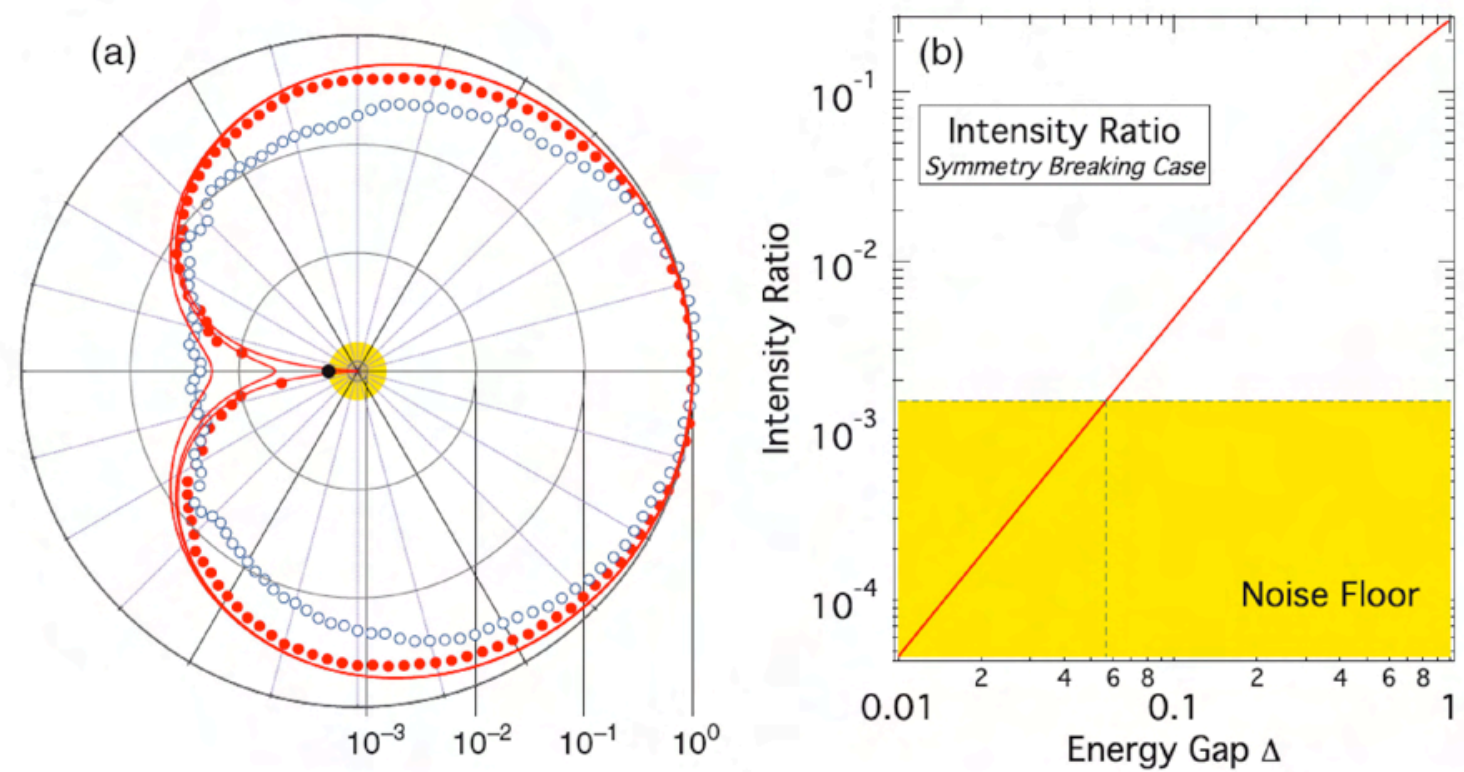

Figure 20: (a) Polar plot of the intensity of the Fermi contours for monolayer (solid circles) and bilayer graphene (open circles), obtained by fitting momentum distribution curves (MDCs) taken along radial cuts through the K point of the Brillouin zone. The intensity scale is logarithmic. Also shown are theoretical intensities for asymmetry parameters $\Delta=0.0,0.1$ and $0.2 \mathrm{eV}$ (solid lines) using Shirley's formalism [93]. The solid black data point is an upper limit based on the noise floor indicated by the central yellow circle. (b) The ratio of the weakest to strongest emission intensities as a function of asymmetry parameter. The noise floor (yellow region) establishes the maximum value of the asymmetry parameter $~ 55$ meV admitted by our measured intensity distribution. From Bostwick et al. [32].

applications, Graphene has been named "the simplest complex material" [32], and is unique in many ways, being truly 2-dimensional, having massless "Dirac Fermion" carriers, and combining properties of metals and semiconductors. Graphene brings together quantum electrodynamics issues and condensed matter physics, opening up new experiments in both fields. A fascinating aspect of research is that graphene can be modified by controlling the number of layers, by chemical doping, external fields, and its geometry. Moreover, graphene can be easily investigated experimentally using transport methods (with a particularly low entry barrier, since its preparation does not require complex laboratory equipment), and the tools of modern surface and interface science such as scanning probe techniques, electron microscopy, electron diffraction, and photoelectron spectroscopy, since it is a true 2D system that is not buried inside a bulk structure [15]. Angle-resolved photoemission has a special role 
to play in the investigation of graphene's electronic structure, as documented in the preceding sections: it is sensitive not only to the valence band energy structure but also its symmetry in $\mathrm{k}$-space, and can give direct information on the many-body interactions; in fact, it is a model system for correlation and many-body interactions for which adequate theories are still under development, and as such is one of the most versatile systems in condensed matter physics.

As documented in this review, the basic aspects of the electronic structure of single and few layer graphene are quite well understood, and can be modeled on the basis of band structure calculations. With respect to many body processes, more work is clearly required, relating to the coupling of quasiparticles to different excitations on the bilayer, the effect of disorder, and how these modify the physical properties oh graphene. A most interesting aspect, and one in which the techniques of surface and interface science are particularly important, is the effect of chemical modification of graphene, i.e. by adsorption, substitution or intercalation. Doping effects may change the chemical potential as discussed above, while adsorbed species that strongly interact with the graphene lattice may lead to hybridization effects that induce a considerable modification in the electronic structure. The introduction of d- and f-metal atoms, for example, may have a strong influence on electron-electron interaction and lead to magnetic effects. An almost uncharted territory experimentally is the effect of confinement in one-dimensional ("nano-ribbons") and zero-dimensional (quantum dots) graphene structures. While the former seem to present some preparation difficulties, the latter exist in the form of large molecules [159] consisting of dozens of benzene rings that may be deposited on suitable substrates. In the context of edge states of low dimensional structures [160], functionalization through ligands of different kinds is another most interesting aspect, which may enhance a possible role of graphene as sensor in chemical environments.

Probably the most important aspect from a materials science and applications-oriented 
point of view is the question of how to prepare large scale epitaxial graphene. Important progress has been made for graphene on silicon carbide [63] and on transition metals [161-163], and chemical means of preparing graphene coatings have been developed $[164,165]$; attempts to achieve large scale growth have recently been reviewed by Geim [8]. An intense research effort is under way to improve these processes and develop new ones, and given the expertise available in the materials science of carbon-based materials rapid progress in this area can be expected. Research into the properties of graphene and its applications will remain a rewarding and exciting field of research for many years to come.

Acknowledgements: Support by the DFG under contract SE 1087/5-1, contract WE 4542-5-1, and within the Cluster of Excellence 'Engineering of Advanced Materials' (www.eam.uni-erlangen.de) at the Friedrich-Alexander-Universität ErlangenNürnberg, by BaCaTeC, and by the BMBF under contract $05 \mathrm{ES} 3 \mathrm{XBA} / 5$. The work carried out at the Advanced Light Source was supported by the Director, Office of Science, Office of Basic Energy Sciences, US Department of Energy under Contract No. DE-AC03-76SF00098. K.H., J.McC. and T.O acknowledge the support by the Max Planck Society. 
References

1. K. S. Novoselov, A. K. Geim, S. V. Morozov, D. Jiang, M. I. Katsnelson, I. V. Grigorieva, S. V. Dubonos and A. A. Firsov, Nature 438, 197-200 (2005).

2. Y. Zhang, Y. W. Tan, H. L. Stormer and P. Kim, Nature 438, 201-4 (2005).

3. C. Berger, Z. Song, X. Li, X. Wu, N. Brown, C. Naud, D. Mayou, T. Li, J. Hass, A. N. Marchenkov, E. H. Conrad, P. N. First and W. A. de Heer, Science 312, 1191-6 (2006).

4. A. K. Geim and K. S. Novoselov, Nat. Mater 6, 183 (2007).

5. A. K. Geim and A. H. MacDonald, Phys. Today 60, 35-41 (2007).

6. M. I. Katsnelson, Materials Today 10, 20-7 (2007).

7. A. C. Neto, F. Guinea and N. M. Peres, Physics World 19, 33-7 (2006).

8. A. K. Geim, Science 324, 1530-4 (2009).

9. P. R. Wallace, Physical Review 71, 622-34 (1947).

10. J. C. Slonczewski and P. R. Weiss, Physical Review 109, 272-9 (1958).

11. G. S. Painter and D. E. Ellis, Phys.Rev. B 1, 4747-52 (1970).

12. D. P. Divincenzo and E. J. Mele, Phys Rev B 29, 1685-94 (1984).

13. G. W. Semenoff, Phys. Rev. Lett 53, 2449-52 (1984).

14. F. D. M. Haldane, Phys. Rev. Lett 61, 2015-8 (1988).

15. A. H. Castro Neto, F. Guinea, N. M. R. Peres, K. S. Novoselov and A. K. Geim, Reviews of Modern Physics 81, 109-62 (2009).

16. R. Saito, G. Dresselhaus and M. S. Dresselhaus, Physical Properties of Carbon Nanotubes (Imperial College Press, 1998), Vol. 38, p. 169-74.

17. L. Landau, Physikalische Zeitschrift der Sowjetunion 11, 545 (1937).

18. N. D. Mermin, N D and H. Wagner, H, Phys. Rev. Lett 17, 1133 (1966).

19. N. D. Mermin, N D, Physical Review 176, 250 (1968).

20. K. S. Novoselov, D. Jiang, F. Schedin, T. J. Booth, V. V. Khotkevich, S. V. Morozov and A. K. Geim, Proc Natl Acad Sci U S A 102, 10451-3 (2005).

21. K. S. Novoselov, A. K. Geim, S. V. Morozov, D. Jiang, Y. Zhang, S. V. Dubonos, I. V. Grigorieva and A. A. Firsov, Science 306, 666-9 (2004).

22. I. Forbeaux, J. M. Themlin and J. M. Debever, Phys.Rev. B 58, 16396-406 (1998).

23. D. Farias, A. M. Shikin, K. H. Rieder and Y. S. Dedkov, J. Phys.: Condens. Matter 11, 8453-8 (1999).

24. C. Berger, Z. M. Song, T. B. Li, X. B. Li, A. Y. Ogbazghi, R. Feng, Z. T. Dai, A. N. Marchenkov, E. H. Conrad, P. N. First and W. A. de Heer, J Phys. Chem. B 108, 19912-6 (2004).

25. T. Seyller, K. V. Emtsev, K. Gao, F. Speck, L. Ley, A. Tadich, L. Broekman, J. D. Riley, R. C. G. Leckey, O. Rader and others, Surf.Sci. 600, 3906-11 (2006).

26. K. S. Novoselov, Z. Jiang, Y. Zhang, S. V. Morozov, H. L. Stormer, U. Zeitler, J. C. Maan, G. S. Boebinger, P. Kim and A. K. Geim, Science 315, 1379- (2007).

27. O. Klein, Z.Physik 53, 157 (1929).

28. M. I. Katsnelson, K. S. Novoselov and A. K. Geim, Nat. Physics 2, 620 (2006).

29. M. I. Katsnelson and K. S. Novoselov, Sol. Stat. Commun. 143, 3-13 (2007).

30. J. W. McClure, Physical Review 104, 666-71 (1956).

31. C. W. J. Beenakker, Reviews of Modern Physics 80, 1337-54 (2008).

32. A. Bostwick, T. Ohta, J. L. McChesney, K. V. Emtsev, T. Seyller, K. Horn and E. Rotenberg, New J.Physics 9, 385 (2007). 
33. W. A. de Heer, C. Berger, X. S. Wu, P. N. First, E. H. Conrad, X. B. Li, T. B. Li, M. Sprinkle, J. Hass, M. L. Sadowski, M. Potemski and G. Martinez, Solid State Commun. 143, 92-100 (2007).

34. A. J. Van Bommel, J. E. Crombeen and A. Van Tooren, Surf.Sci. 48, 463-72 (1975).

35. C. Berger, Z. Song, T. Li, X. Li, A. Y. Ogbazghi and R. Feng, J.Phys.Chem. B 108, 19912 (2004).

36. P. Blake, E. W. Hill, A. H. C. Neto, K. S. Novoselov, D. Jiang, R. Yang, T. J. Booth and A. K. Geim, Appl. Phys. Lett. 91, (2007).

37. J. C. Meyer, A. K. Geim, M. I. Katsnelson, K. S. Novoselov, T. J. Booth and S. Roth, Nature 446, 60-3 (2007).

38. A. Nagashima, K. Nuka, H. Itoh, T. Ichinokawa, C. Oshima and S. Otani, Surf.Sci. 291, 93-8 (1993).

39. A. Nagashima, N. Tejima and C. Oshima, Physical Review. B, Condensed matter 50, 17487-95 (1994).

40. A. M. Shikin, G. V. Prudnikova, V. K. Adamchuk, F. Moresco and K. H. Rieder, Phys.Rev. B 62, 13202-8 (2000).

41. Y. S. Dedkov, A. M. Shikin, V. K. Adamchuk, S. L. Molodtsov, C. Laubschat, A. Bauer and G. Kaindl, Phys. Rev. B 64, 35405 (2001).

42. H. Yanagisawa, T. Tanaka, Y. Ishida, M. Matsue, E. Rokuta, S. Otani and C. Oshima, Surf. Int. An. 37, 133-6 (2005).

43. T. Fujita, W. Kobayashi and C. Oshima, Surf. Int. An. 37, 120-3 (2005).

44. J. Coraux, A. T. N'Diaye, C. Busse and T. Michely, Nano Lett 8, 565-70 (2008).

45. I. Pletikosic, M. Kralj, P. Pervan, R. Brako, J. Coraux, A. T. N'Diaye, C. Busse and T. Michely, Phys. Rev. Lett 102, (2009).

46. L. Muehlhoff, W. J. Choyke, M. J. Bozack and J. T. Yates Jr, Journal of Applied Physics 60, 2842 (1986).

47. T. Seyller, A. Bostwick, K. V. Emtsev, K. Horn, L. Ley, J. L. McChesney, T. Ohta, J. D. Riley, E. Rotenberg and F. Speck, phys. stat. sol. B 245, 1436-46 (2008).

48. P. Avouris, Z. Chen and V. Perebeinos, Nat. Nanotechnol 2, 605-15 (2007).

49. Y. M. Lin, K. A. Jenkins, A. Valdes-Garcia, J. P. Small, D. B. Farmer and P. Avouris, Nano Lett 9, 422-6 (2009).

50. J. S. Bunch, A. M. van der Zande, S. S. Verbridge, I. W. Frank, D. M. Tanenbaum, J. M. Parpia, H. G. Craighead and P. L. McEuen, Science 315, 490-3 (2007).

51. L. I. Johansson, P. A. Glans and N. Hellgren, Surf.Sci. 405, 288-97 (1998).

52. T. Ohta, A. Bostwick, T. Seyller, K. Horn and E. Rotenberg, Science 313, 951-4 (2006).

53. A. Bostwick, T. Ohta, T. Seyller, K. Horn and E. Rotenberg, Nat. Physics 3, 36-40 (2007).

54. T. Ohta, A. Bostwick, J. L. McChesney, T. Seyller, K. Horn and E. Rotenberg, Phys. Rev. Lett 98, 206802 (2007).

55. K. V. Emtsev, T. Seyller, F. Speck, L. Ley, P. Stojanov, J. D. Riley and R. G. C. Leckey, Materials Science Forum 556-557, 525-8 (2007).

56. (http://web.mit.eud/invent/iow/acheson.html, The Lemelson - MIT Program, Edward Acheson Carborundum, electronic source .

57. L. I. Johansson, F. Owman and P. Mårtensson, Phys.Rev. B 53, 13793-802 (1996).

58. U. Starke, J. Schardt and M. Franke, Applied Physics A: Materials Science \& Processing 65, 587-96 (1997).

59. U. Starke, M. Franke, J. Bernhardt, J. Schardt, R. Reuter and K. Heinz, Materials Science Forum 312 (1998).

60. T. Seyller, Applied Physics A: Materials Science \& Processing 85, 371-85 (2006). 
61. J. Hass, F. Varchon, J. E. Millán-Otoya, M. Sprinkle, N. Sharma, W. A. de Heer, C. Berger, P. N. First, L. Magaud and E. H. Conrad, Phys. Rev. Lett 100, 125504 (2008).

62. I. Forbeaux, J. M. Themlin, A. Charrier, F. Thibaudau and J. M. Debever, Appl. Surf. Sci 162, 406-12 (2000).

63. K. V. Emtsev, A. Bostwick, K. Horn, J. Jobst, G. L. Kellogg, L. Ley, J. L. McChesney, T. Ohta, S. A. Reshanov, J. Röhrl, E. Rotenberg, A. K. Schmid, D. Waldmann, H. B. Weber and T. Seyller, Nat. Mater 8, 203-7 (2009).

64. E. Rollings, G. H. Gweon, S. Y. Zhou, B. S. Mun, J. L. McChesney, B. S. Hussain, A. V. Fedorov, P. N. First, W. A. de Heer and A. Lanzara, Journal of Physics and Chemistry of Solids 67, 2172-7 (2006).

65. J. Bernhardt, J. Schardt, U. Starke and K. Heinz, Appl. Phys. Lett. 74, 1084 (1999).

66. U. Starke, in Recent Major Advances in SiC, edited by Choyke, W J, Matsunami, H and Pensl, G (Springer-Verlag, 2004) p. 281.

67. A. Bostwick, K. V. Emtsev, K. Horn, E. Huwald, L. Ley, J. L. McChesney, T. Ohta, J. Riley, E. Rotenberg, F. Speck and T. Seyller, Adv. Solid State Phys. 47, 159-70 (2008).

68. P. Mallet, F. Varchon, C. Naud, L. Magaud, C. Berger and J. Y. Veuillen, Phys.Rev. B 76, 41403 (2007).

69. K. V. Emtsev, F. Speck, T. Seyller, L. Ley and J. D. Riley, Phys.Rev. B 77, 155303 (2008).

70. V. W. Brar, Y. Zhang, Y. Yayon, T. Ohta, J. L. McChesney, A. Bostwick, E. Rotenberg, K. Horn and M. F. Crommie, Appl. Phys. Lett. 91, 122102 (2007).

71. M. H. Tsai, C. S. Chang, J. D. Dow and I. S. T. Tsong, Phys.Rev. B 45, 1327-32 (1992).

72. J. E. Northrup and J. Neugebauer, Phys. Rev. B 52, 17001-4 (1995).

73. V. Van Elsbergen, T. U. Kampen and W. Monch, Surf.Sci. 365, 443-52 (1996).

74. C. Riedl, U. Starke, J. Bernhardt, M. Franke and K. Heinz, Phys.Rev. B 76, 245406 (2007).

75. W. Chen, H. Xu, L. Liu, X. Gao, D. Qi, G. Peng, S. C. Tan, Y. Feng, K. P. Loh and A. T. S. Wee, Surf.Sci. 596, 176-86 (2005).

76. P. Lauffer, K. V. Emtsev, R. Graupner, T. Seyller, L. Ley, S. A. Reshanov and H. B. Weber, Phys.Rev. B 77, 155426 (2008).

77. K. Horn, in Handbook of Surface Science (Elsevier Science, Amsterdam, 2000).

78. T. Seyller, K. V. Emtsev, F. Speck, K. Y. Gao and L. Ley, Appl. Phys. Lett. 88, 242103 (2006).

79. E. J. Duplock, M. Scheffler and P. J. D. Lindan, Phys. Rev. Lett 92, 225502 (2004).

80. H. Hibino, H. Kageshima, F. Maeda, M. Nagase, Y. Kobayashi and H. Yamaguchi, Phys.Rev. B 77, 075413 (2008).

81. T. Ohta, F. El Gabaly, A. Bostwick, J. McChesney, K. V. Emtsev, A. K. Schmid, T. Seyller, K. Horn and E. Rotenberg, New J.Physics 10, 023034 (2008).

82. H. Hibino, H. Kageshima, F. Maeda, M. Nagase, Y. Kobayashi and H. Yamaguchi, e-Journal of Surface Science and Nanotechnology 6, 107-10 (2008).

83. J. B. Hannon and R. M. Tromp, Phys Rev B 77, 241404 (1975).

84. R. M. Tromp and J. B. Hannon, Phys. Rev. Lett 102, (2009).

85. I. Langmuir I, Phys.Rev. (Series I) 34, 401 (1912).

86. G. R. Fonda, Physical Review 31, 260-6 (1928).

87. G. Beamson, D. Briggs, S. F. Davies, I. W. Fletcher, D. T. Clark, J. Howard, U. Gelius, B. Wannberg and P. Balzer, Surf. Int. An. 15, (1990).

88. N. Martensson, P. Baltzer, P. A. Bruhwiler, J. O. Forsell, A. Nilsson, A. Stenborg and B. Wannberg, J.Elec.Spec.Relat.Phenom. 70, 117-28 (1995).

89. A. Bostwick, T. Ohta, J. L. McChesney, T. Seyller, K. Horn and E. Rotenberg, Eur Phys J-Spec 
Top 148, 5-13 (2007).

90. S. Reich, J. Maultzsch, C. Thomsen and P. Ordejón, Phys.Rev. B 66, 35412 (2002).

91. C. Heske, R. Treusch, F. J. Himpsel, S. Kakar, L. J. Terminello, H. J. Weyer and E. L. Shirley, Phys.Rev. B 59, 4680-4 (1999).

92. E. L. Shirley, L. J. Terminello, A. Santoni and F. J. Himpsel, Phys.Rev. B 51, 13614-22 (1995).

93. T. C. Chiang, Surface Science Reports 39, 181 (2000).

94. L. Aballe, C. Rogero, S. Gokhale, S. Kulkarni and K. Horn, Surf.Sci. 482, 488-94 (2001).

95. E. McCann, Phys.Rev. B 74, 161403 (2006).

96. F. Guinea, A. H. Castro Neto and N. M. R. Peres, Phys.Rev. B 73, 245426 (2006).

97. G. Moos, C. Gahl, R. Fasel, M. Wolf and T. Hertel, Phys. Rev. Lett 87, 267402 (2001).

98. K. Sugawara, T. Sato, S. Souma, T. Takahashi and H. Suematsu, Phys.Rev. B 73, 45124 (2006).

99. L. Pietronero, S. Strässler, H. R. Zeller and M. J. Rice, Phys. Rev. Lett 41, $763-7$ (1978).

100. S. V. Morozov, K. S. Novoselov, F. Schedin, D. Jiang, A. A. Firsov and A. K. Geim, Phys.Rev. B 72, 201401 (2005).

101. A. Mugarza, J. E. Ortega, F. J. Himpsel and F. J. Garcia de Abajo, Phys.Rev. B 67, 81404 (2003).

102. C. Didiot, Y. Fagot-Revurat, S. Pons, B. Kierren, C. Chatelain and D. Malterre, Phys.Rev. B 74, 81404 (2006).

103. R. Hoffman, Solids and Surfaces: A Chemist's View of Bonding in Extended Structures (VCH Weinheim 1988, 1988).

104. S. Y. Zhou, G. H. Gweon and A. Lanzara, Ann. Phys. 321, 1730-46 (2006).

105. S. G. Louie, P. Thiry, R. Pinchaux, Y. Pétroff, D. Chandesris and J. Lecante, Phys. Rev. Lett 44, 549-53 (1980).

106. P. Hofmann, C. Søndergaard, S. Agergaard, S. V. Hoffmann, J. E. Gayone, G. Zampieri, S. Lizzit and A. Baraldi, Phys.Rev. B 66, 245422 (2002).

107. J. Lobo and A. Mascaraque, Journal of Physics: Condensed Matter 18, L395-400 (2006).

108. K. S. Novoselov, E. McCann, S. V. Morozov, V. I. Fal'ko, M. I. Katsnelson, U. Zeitler, D. Jiang, F. Schedin and A. K. Geim, Nat. Physics 2, 177-80 (2006).

109. E. McCann and V. I. Fal'ko, Phys. Rev. Lett 96, 086805 (2006).

110. Y. B. Zhang, Y. W. Tan, H. L. Stormer and P. Kim, Nature 438, 201-4 (2005).

111. E. V. Gorbar, V. P. Gusynin, V. A. Miransky and I. A. Shovkovy, Phys.Rev. B 66, 45108 (2002).

112. Y. Zheng and T. Ando, Phys.Rev. B 65, 245420 (2002).

113. J. C. Charlier, X. Gonze and J. P. Michenaud, Phys.Rev. B 43, 4579-89 (1991).

114. X. Blase, A. Rubio, S. G. Louie and M. L. Cohen, Phys. Rev. B 51, 6868-75 (1995).

115. Y. Zhang, J. P. Small, W. V. Pontius and P. Kim, Appl. Phys. Lett. 86, 073104 (2005).

116. T. Kihlgren, T. Balasubramanian, L. Walldén and R. Yakimova, Surf.Sci. 600, 1160-4 (2006).

117. J. B. Oostinga, H. B. Heersche, X. Liu, A. F. Morpurgo and L. M. Vandersypen, Nat. Mater 7, 151-7 (2008).

118. I. Gierz, C. Riedl, U. Starke, C. R. Ast and K. Kern, Nano Lett 8, 4603-7 (2008).

119. W. Chen, S. Chen, D. C. Qi, X. Y. Gao and A. T. Wee, J Am Chem Soc 129, 10418-22 (2007).

120. S. Y. Zhou, D. A. Siegel, A. V. Fedorov and A. Lanzara, Phys. Rev. Lett 101, 086402 (2008).

121. T. O. Wehling, K. S. Novoselov, S. V. Morozov, E. E. Vdovin, M. I. Katsnelson, A. K. Geim and A. I. Lichtenstein, Nano Lett 8, 173-7 (2008).

122. F. Schedin, A. K. Geim, S. V. Morozov, E. W. Hill, P. Blake, M. I. Katsnelson and K. S.

Novoselov, Nat. Mater 6, 652-5 (2007). 
123. A. Mattausch and O. Pankratov, Phys. Rev. Lett 99, (2007).

124. Z. K. Tang, L. Zhang, N. Wang, X. X. Zhang, G. H. Wen, G. D. Li, J. N. Wang, C. T. Chan and P. Sheng, Science 292, 2462-5 (2001).

125. M. Kociak, A. Y. Kasumov, S. Guéron, B. Reulet, I. I. Khodos, Y. B. Gorbatov, V. T. Volkov, L. Vaccarini and H. Bouchiat, Phys. Rev. Lett 86, 2416-9 (2001).

126. T. E. Weller, M. Ellerby, S. S. Saxena, R. P. Smith and N. T. Skipper, Nat. Physics 1, 39-41 (2005).

127. T. Valla, A. V. Fedorov, P. D. Johnson and S. L. Hulbert, Phys. Rev. Lett 83, 2085-8 (1999).

128. Grimvall, The electron-phonon coupling in metals (North-Holland, Amsterdam, 1981).

129. J. Schaefer J, D. Schrupp D, E. Rotenberg E, S. D. Kevan S D and R. Claessen R, Appl. Phys. A 80, (2005).

130. Hengsberger, M, Purdie, D, Segovia, P, Garnier, M and Baer,Y, Phys. Rev. Lett 83, 592.

131. T. Valla, A. V. Fedorov, P. D. Johnson, B. O. Wells, S. L. Hulbert, Q. Li, G. D. Gu and N. Koshizuka, Science 285, 2110-3 (1999).

132. A. Damascelli, Z. Hussain and Z. X. Shen, Rev. Mod. Phys. 75, 473-541 (2003).

133. A. Kaminski and H. M. Fretwell, New J.Physics 7, - (2005).

134. A. Bostwick, T. Ohta, J. L. McChesney, T. Seyller, K. Horn and E. Rotenberg, Sol. Stat. Commun. 143, 63-71 (2007).

135. S. Xu, J. Cao, C. C. Miller, D. A. Mantell, R. J. D. Miller and Y. Gao, Phys. Rev. Lett 76, 483-6 (1996).

136. S. Xu, J. Cao, C. C. Miller, D. A. Mantell, R. J. D. Miller and Y. Gao, Phys. Rev. Lett 77, 1411(1996).

137. H. Frohlich, Journal of Physics C 1, 544 (1968).

138. J. Ruvalds, F. Brosens, L. F. Lemmens and J. T. Devreese, Sol. Stat. Commun. 23, 243-6 (1977).

139. M. Mucha-Kruczynski, O. Tsyplyatyev, A. Grishin, E. McCann, V. I. Fal'ko, A. Bostwick and E. Rotenberg, Phys.Rev. B 77, 195403 (2008).

140. K. Sugawara, T. Sato, S. Souma, T. Takahashi and H. Suematsu, Phys. Rev. Lett 98, 36801 (2007).

141. Kordyuk, A A, Borisenke, S V, Koitzsch, A, Fink J, Knupfer M and Berger H, Phys.Rev. B 71, 214513 (2005).

142. E. Rotenberg, E, J. Schaefer, J and S. D. Kevan, S D, Phys. Rev. Lett 84, 2925.

143. L. Vitali, M. A. Schneider, K. Kern, L. Wirtz and A. Rubio, Physical Review-Section BCondensed Matter 69, 121414 (2004).

144. C. Calandra, C and F. Mauri, F, Phys. Rev. Lett 95, 237002 (2005).

145. A. H. Castro Neto, Phys. Rev. Lett 86, 4382-5 (2001).

146. G. F. Giuliani and J. J. Quinn, Phys. Rev. B 26, $4421-8$ (1982).

147. P. Hawrylak, G. Eliasson and J. J. Quinn, Phys. Rev. B 37, 10187-94 (1988).

148. J. González, F. Guinea and M. A. Vozmediano, Phys. Rev. Lett 77, 3589-92 (1996).

149. L. Zheng and Das Sarma S, Phys. Rev. Lett 77, 1410 (1996).

150. S. Xu, S, J. Cao, J, C. C. Miller, C C, D. A. Mantell, D A, R. J. D. Miller, R J D and Y. Gao, Y, Phys. Rev. Lett 77, 1411.

151. J. H. Ho, C. P. Chang, R. B. Chen and M. F. Lin, Physics Letters A 357, 401-6 (2006).

152. C. H. Park, F. Giustino, C. D. Spataru, M. L. Cohen and S. G. Louie, Phys. Rev. Lett 102, (2009).

153. S. Y. Zhou, G. H. Gweon, A. V. Fedorov, P. N. First, W. A. de Heer, D. H. Lee, F. Guinea, A. H. Castro Neto and A. Lanzara, Nat. Mater 6, 770-5 (2007). 
154. E. Rotenberg, A. Bostwick, T. Ohta, J. L. McChesney, T. Seyller and K. Horn, Nat. Mater 7, 258-9 (2008).

155. F. Varchon, R. Feng, J. Hass, X. Li, B. N. Nguyen, C. Naud, P. Mallet, J. Y. Veuillen, C. Berger, E. H. Conrad and others, Phys. Rev. Lett 99, 126805 (2007).

156. A. Mattausch and O. Pankratov, Phys. Rev. Lett 99, (2007).

157. T. Ando and T. Nakanishi, J.Phys.Soc. Jpn. 67, 1704-13 (1998).

158. T. Ando, T. Nakanishi and R. Saito, J.Phys.Soc. Jpn. 67, 2857-62 (1998).

159. L. Zhi and K. Müllen, Journal of Materials Chemistry 18, 1472-84 (2008).

160. Y. W. Son, M. L. Cohen and S. G. Louie, Nature 444, 347-9 (2006).

161. P. W. Sutter, J. I. Flege and E. A. Sutter, Nat. Mater 7, 406-11 (2008).

162. K. S. Kim, Y. Zhao, H. Jang, S. Y. Lee, J. M. Kim, K. S. Kim, J. H. Ahn, P. Kim, J. Y. Choi and B. H. Hong, Nature 457, 706-10 (2009).

163. A. Reina, X. Jia, J. Ho, D. Nezich, H. Son, V. Bulovic, M. S. Dresselhaus and J. Kong, Nano Lett 9, 30-5 (2009).

164. X. Li, G. Zhang, X. Bai, X. Sun, X. Wang, E. Wang and H. Dai, Nat. Nanotechnol 3, 538-42 (2008).

165. Y. Hernandez, V. Nicolosi, M. Lotya, F. M. Blighe, Z. Sun, S. De, I. T. McGovern, B. Holland, M. Byrne, Y. K. Gun'ko, J. J. Boland, P. Niraj, G. Duesberg, S. Krishnamurthy, R. Goodhue, J. Hutchison, V. Scardaci, A. C. Ferrari and J. N. Coleman, Nat. Nanotechnol 3, 563-8 (2008). 